\title{
NEUROPSY CHOLOGICAL EVIDENCE FOR A TOPOGRAPHICAL LEARNING MECHANISM IN PARAHIPPOCAMPAL CORTEX
}

\author{
Russell Epstein \\ Medical Research Council Cognition and Brain Sciences Unit, Cambridge, UK and MIT Department of Brain and Cognitive Sciences, \\ Cambridge, USA
}

Edgar A. DeYoe

Medical College of Wisconsin, Milwaukee, USA

Daniel Z. Press

Beth Israel Deaconess Medical Center, Boston, USA

Allyson C. Rosen

Stanford University, Stanford, USA

Nancy Kanwisher

MIT Department of Brain and Cognitive Sciences, Cambridge, USA

\begin{abstract}
The Parahippocampal Place Area (PPA; Epstein \& Kanwisher, 1998) is a region within posterior parahippocampal cortex that responds selectively to visual stimuli that convey information about the layout of local space. Here we describe two patients who suffered damage to the PPA after vascular incidents. Both subsequently exhibited memory problems for topographical materials and were unable to navigate unassisted in unfamiliar environments. Performance on a continuous n-back visual memory test was significantly lower for novel scene-like stimuli than for novel object-like stimuli. In contrast, performance was normal on a famous landmark recognition task and on two perceptual tasks that required on-line analysis of scene geometry. Both patients were able to produce accurate maps of premorbidly learned places but were unable to produce accurate maps of new places. These results converge with previous neuroimaging work to demonstrate that the PPA (1) is selectively involved in processing information about the geometry of surrounding space, and (2) may play a more critical role in the encoding of this information into memory than in the initial perceptual processing, recognition, or recall of this information.
\end{abstract}

Requests for reprints should be addressed to Russell Epstein, MRC Cognition \& Brain Sciences Unit, 15 Chaucer Road, Cambridge CB2 2EF, UK (Tel: +44 (0) 1223-355-294, ext. 800; Fax:+44 (0) 1223-359-062; Email: russell.epstein@mrc-cbu.cam.ac.uk).

We thank Damian Stanley and Alison Harris for assistance with these experiments, Stephan Heckers and Facundo Manes for anatomical advice, Kim Graham for comments on the manuscript, Tom Hammeke for advice about neuropsychological tests, Matthew Brett for assistance with MRI normalisation, and Marvin Chun, Frances Wang, and Liz Spelke for helpful discussions. In addition, we would also like to express our thanks to GR and $\mathrm{CO}$ for their cheerful and enthusiastic participation in this research. This research was supported by NIMH postdoctoral fellowship MH11459 to RE, NEI and NIMH grants EY10244 and MH51358 to EAD, and NIMH grant MH56037 to NK. 


\section{INTRODUCTION}

All mobile organisms must solve the fundamental problem of navigation. One way to gain insight into the cognitive and neural mechanisms underlying navigation is to investigate the functional organisation of the brain areas involved. Research on animals and humans has suggested that a number of regions play a role, including the parietal lobes (Stark, Coslett, \& Saffran, 1996), retrosplenial cortex (Chen, Lin, Green, Barnes, \& McNaughton, 1994), hippocampus (Maguire et al., 1998; O’Keefe \& Nadel, 1978), and parahippocampal cortex (Aguirre, Detre, Alsop, \& D'Esposito, 1996; Bohbot et al., 1998). Here we focus on parahippocampal cortex. We describe two patients who suffered damage to this region of cortex after vascular incidents. Both subsequently developed severe deficits in the topographical domain, which we define as the domain of information relevant to spatial navigation.

Previous reports in the neuropsychological literature have described patients with "topographical disorientation," who are unable to successfully find their way through locomotor space but do not exhibit general cognitive or memory impairments (see Aguirre \& D'Esposito, 1999, for review). The nature of the specific problem varies from patient to patient. For example, some patients show an inability to understand spatial relationships between different locations (Levine, Warach, \& Farah, 1985, patient 2), whereas others are unable to recognise navigationally relevant stimuli such as landmarks but can accurately describe routes between the places they cannot identify (Pallis, 1955). Patients also differ in their relative ability to handle novel vs. familiar topographical information. Some are able to navigate through familiar environments or recall old topographical information but cannot learn novel environments (Habib \& Sirigu, 1987, patients 1 and 2; Ross, 1980; Teng \& Squire, 1999), whereas others are impaired at both learning of new materials and recognition/recall of old materials (Habib \& Sirigu, 1987, patients 3 and 4; Incisa della Rocchetta, Cipolotti, \& Warrington, 1996; Landis, Cummings, Benson, \& Palmer, 1986;
McCarthy, Evans, \& Hodges, 1996; Whiteley \& Warrington, 1978).

Based on comprehensive review of the topographical disorientation literature, Aguirre and D'Esposito (1999) argued that the lingualparahippocampal region is one of several areas that are critically involved in navigation. Damage to this region often results in an inability to use salient topographical features such as landscapes and buildings to orient oneself ("landmark agnosia," e.g., Landis et al., 1986; Pallis, 1955) or in a more general inability to learn new topographical information ("anterograde disorientation," e.g., Habib \& Sirigu, 1987, patients 1 and 2). In a recent study of 127 patients with focal lesions, Barrash, Damasio, Adolphs, and Tranel (2000) observed that patients with damage to this region almost always displayed severe impairment on a real-world route learning task. Furthermore, Bohbot et al. (1998) found that patients with right-hemisphere parahippocampal cortex lesions were impaired at a human analogue of the Morris water maze task in which they were required to remember the location of a hidden floor platform within a room (a deficit not found in patients whose damage was restricted to the hippocampus proper).

Neuroimaging studies have also implicated the lingual-parahippocampal region in navigation. Several studies have examined brain activity in normal subjects during performance of a number of navigational tasks, including navigation through a virtual reality environment (Aguirre et al., 1996), watching videotapes depicting navigation through a real environment (Maguire, Frackowiak, \& Frith, 1997), and mentally imagining navigation through a real environment (Ghaem et al., 1997; Maguire et al., 1998). All of these studies found greater activation in the parahippocampal region (and sometimes other medial temporal regions as well, Ghaem et al., 1997; Maguire et al., 1997,1998) when the navigation task was compared to a less navigationally demanding control task.

What role does parahippocampal cortex play in these navigational tasks? In earlier work, we addressed this question by using fMRI to examine neural activity in parahippocampal cortex and other 
brain regions while subjects viewed a wide variety of visual stimuli (Epstein \& Kanwisher, 1998). We found that a region abutting the collateral sulcus near the parahippocampal-lingual boundary responded significantly more strongly when subjects viewed navigationally relevant stimuli such as street scenes, landscapes, or buildings than when they viewed other kinds of visual stimuli such as faces, objects, or scrambled scenes ${ }^{1}$. This response was found even when subjects simply watched the stimuli passively and were not required to perform any navigational task. We named this region the "parahippocampal place area," or PPA, because it responded strongly to depictions of places. The PPA appears to overlap with the regions activated in previous neuroimaging studies of navigation (e.g., Aguirre et al., 1996).

A critical determinant of PPA activation appears to be the presence in the stimulus of information about the shape or layout of the immediate environment. The PPA responds no more strongly to photographs of rooms filled with furniture and objects than it does to photographs of the same rooms empty of all objects (i.e., just bare walls). It also responds much more strongly to "scenes" made out of Lego blocks (which have a geometric structure similar to a room or street scene) than it does to objects made out of the same Lego materials (Epstein, Harris, Stanley, \& Kanwisher, 1999). Thus, the PPA is sensitive to the presence of a specific kind of geometric organisation in the stimulus even if the stimulus does not depict a real place in the world. In particular, it responds more strongly to depictions of surfaces that in some sense "enclose" the observer and define a space within which one can act (i.e., the walls of a room or the side of the distant hill) than to depictions of surfaces that define objects that the observer can act upon. These results are generally consistent with behavioural results from rats (Cheng, 1986; Gallistel, 1990; Margules \& Gallistel, 1988) and human infants (Hermer \& Spelke, 1994, 1996; HermerVazquez, Spelke, \& Katsnelson, 1999) that suggest that information about the geometry of surrounding space - the "lay of the land"-plays a privileged role in orientation and navigation.

What specific cognitive task does the PPA perform? Given the fact that landmark agnosia often results from parahippocampal-lingual damage (Landis et al., 1986), one might hypothesise that the primary role of the PPA is place recognition: identification of familiar locations based on their appearance and/or geometric structure. If so, one might expect the PPA to be sensitive to the novelty/ familiarity of the place depicted in the stimulus. However, we found that the PPA responds just as strongly to photographs of unfamiliar places (i.e., places the subjects had never visited) as it does to photographs of familiar places (Epstein et al., 1999, Expt. 1). In contrast, the PPA is sensitive to the novelty/familiarity of the stimulus itself, responding more strongly to new photographs than to photographs viewed many times previously even when all the photographs depict unfamiliar places (Epstein et al., 1999, Expt. 4). From these results, we inferred that the PPA is more involved in encoding a representation of the spatial structure of the current scene than in relating that scene to one's stored cognitive map of the environment as a whole. However, our data did not allow us to determine whether the PPA activation reflects perceptual encoding, memory encoding, or both.

The purpose of the present report was to more precisely determine the function of the PPA. To this end, we tested the ability of our two patients to perceive, learn, and recognise a variety of visual materials. We predicted that the patients would be more impaired at processing scene-like stimuli (i.e., stimuli that depict spaces that one can navigate through or act within) than object-like stimuli. By using several different kinds of tests, we aimed to determine whether any such selective impairment

\footnotetext{
1 Although full scenes activate the PPA more strongly than do any other kind of visual stimulus, we have also observed higher activity in the PPA when subjects view buildings than when they view other kinds of objects (Epstein et al., 1999), consistent with Aguirre, Zarahn, and D'Esposito's (1998) finding of a significant response to buildings in an adjoining and possibly overlapping region of the anterior lingual gyrus.
} 
was in the perceptual, recognition, or memory domain. To anticipate, we did find evidence for a deficit for visual materials that conveyed information about the shape of surrounding space. This deficit manifested itself during a memory encoding test but not during perceptual tests or a recognition test. Thus, our data suggest that the PPA is necessary for the encoding of spatial layout information into memory, but may not be necessary for many aspects of the initial perceptual analysis, recognition, or recall of this information. We argue that the PPA may be a learning mechanism specifically dedicated to encoding topographical materials into memory.

\section{CASE DESCRIPTIONS AND NEUROPSYCHOLOGICAL TESTING}

In this section, we present the case histories of the two patients in order to provide a general context for the more specific experimental tests reported in the Experimental Investigations section. The patients were chosen for investigation because clinical MRIs indicated that they had suffered damage to the parahippocampal region. This section is divided into four subsections. First, we present a general overview of the case histories. Second, we report the results of some ad hoc tests that give a general indication of the patient's navigational abilities. Third, we discuss other cognitive abilities, including face recognition and episodic memory. Fourth, we report the results of a battery of formal neuropsychological tests performed on the patients. Table 1 summarises relevant observed and selfreported behaviour, and Table 2 summarises the results of the neuropsychological tests.

\section{Overview}

\section{Case 1: GR}

$\mathrm{GR}^{2}$ is a right-handed male with 18 years of education. He was 60 years old at the time of testing. Two-and-a-half years prior to testing, he suffered a right occipital-temporal stroke during cardiac surgery. A second stroke affecting the left occipitaltemporal region occurred a week later. These strokes left him with a number of deficits, including limitation of his peripheral vision (left hemianopsia, right upper quadrantanopsia), dyschromatopsia (details reported in Beauchamp et al., 2000), and topographical disorientation. We

Table 1. Summary of observed and self-reported behaviour

$$
G R
$$

CO

Real-world topographical learning

Map drawing

Scene perception

Face recognition

Episodic memory
Unable to recognise 7/14 newly learned MIT landmarks, despite extensive training regime.

Unable to find way back to lab unassisted, despite extended experience with environment.

Could not draw map of lab; maps of other newly learned environments were inaccurate.

Drew accurate map of premorbid environment (former home).

Reports difficulty understanding the globality of complex scenes.

Some prosopagnosia.

Largely intact, though some difficulties were reported.

Can follow the plot of a movie or novel and report previous day's activities.
Unable to learn 3-room videogame environment.

Able to learn a simple route out of building after many visits.

Map of lab was sketchy, but not entirely inaccurate.

Drew accurate map of premorbid environment (current/former home).

Does not report a perceptual deficit.

Severe prosopagnosia.

Moderate difficulties were reported.

Reports some difficulty following the plot of a book. Accurately reported contents of previous day's newspaper.

${ }^{2}$ GR's true initials are KG (c.f., Beauchamp, Haxby, Rosen, \&DeYoe, 2000), and CO's true initials are KC. We use GR and CO here to avoid confusion between the two patients. 
Table 2. Summary of neuropsychological tests (\% indicates percentile scores)

\begin{tabular}{|c|c|c|}
\hline & $G R$ & $C O$ \\
\hline$I Q$ & $122(93 \%$, WAIS-R) & 134 (99\%, WAIS-III) \\
\hline \multicolumn{3}{|l|}{$W M S-I I I$} \\
\hline Logical memory & $\begin{array}{l}25 \% \text { immediate recall } \\
50 \% \text { delayed recall }\end{array}$ & $\begin{array}{l}27 \% \text { immediate recall } \\
48 \% \text { delayed recall(WMS-R) }\end{array}$ \\
\hline Face memory & $\begin{array}{l}9 \% \text { immediate recall } \\
16 \% \text { delayed recall }\end{array}$ & $\begin{array}{l}16 \% \text { immediate recall } \\
9 \% \text { delayed recall }\end{array}$ \\
\hline Family pictures & $\begin{array}{l}2 \% \text { immediate recall } \\
5 \% \text { delayed recall }\end{array}$ & $\begin{array}{l}<1 \% \text { immediate recall } \\
<1 \% \text { delayed recall }\end{array}$ \\
\hline $\begin{array}{l}\text { Rey Auditory Verbal } \\
\text { Learning test }\end{array}$ & $\begin{array}{l}63 \% \text { immediate recall } \\
14 \% \text { after interference list } \\
16 \% \text { delayed recall }\end{array}$ & $\begin{array}{l}45 \% \text { immediate recall } \\
<1 \% \text { after interference list } \\
3 \% \text { delayed recall }\end{array}$ \\
\hline $\begin{array}{l}\text { Warrington Recognition } \\
\text { Memory tests }\end{array}$ & & $\begin{array}{l}50 \% \text { words } \\
29 \% \text { scenes } \\
<5 \% \text { faces }\end{array}$ \\
\hline 7/24 Spatial Recall & $\begin{array}{l}66 \% \text { immediate recall } \\
2 \% \text { delayed recall }\end{array}$ & \\
\hline Benton Face Recognition & $42 \%$ & $<1 \%$ \\
\hline Memory span & & $\begin{array}{l}\text { Digits: } \\
\text { 99\% forward } \\
99 \% \text { backward } \\
\text { Spatial: } \\
\text { 37\% forward } \\
50 \% \text { backward }\end{array}$ \\
\hline Boston Naming test & Ceiling & Ceiling \\
\hline
\end{tabular}

tested him over a period of 5 days in August 1998, with 3 days of follow-up testing 3 weeks later, and 1 day of additional tests in May 1999. During this time, he was alert and cooperative. Emotional affect and language use was normal. He interacted normally in nonclinical social situations, and his conversation was appropriate and varied. $\mathrm{He}$ remembered the events of previous days and recounted them accurately.

In contrast to this high level of nontopographical functioning, GR demonstrated a dramatic inability to orient himself in space. For example, even after several days of testing, he never learned the relative locations of the different rooms of the lab. According to both GR and his wife, this inability to learn new topographical information was typical of his experience since his strokes, as he frequently gets lost in his daily life. Soon after his injury, he moved to a neighbourhood with many similar-looking houses. He reported that in order to find his new house after a walk to a market six to seven blocks away, he had to rely on street signs to guide him to the correct block, and then examine each house on the block in detail until he could recognise some feature that distinguished his home uniquely. Subsequently, GR and his wife moved to a different house in a different country. He reported that for the first 6 months after the move, his new home was like a "haunted house" for him insofar as he was unable to learn his way around it. For example, he would repeatedly forget that the bedroom had a balcony, and he would be surprised by this fact every time he rediscovered it. After several months, however, he was able to learn the topography of the house sufficiently well to navigate through it (but see below). 


\section{Case 2: $C O$}

$\mathrm{CO}$ is a left-handed male with 20 years of education. He was 60 years old at the time of testing. Two years prior to testing he suffered a right posterior cerebral artery stroke. He first noticed on a weekend that his vision appeared blurred on the left and that he had a tendency to bump into objects on this side. On Monday morning, he was able to successfully negotiate the route to his workplace, a trip that involved a bus ride followed by two subways and then a shuttle to his office building. However, once he entered the building he found himself completely disoriented, unsure of its layout and where his office was located. Interestingly, the company he worked for had moved a few weeks earlier. Although he had managed to arrive at this new building (which was a block away from the previous location), he became lost upon entering it. His neurologic exam revealed a left homonymous hemianopia but no evidence of neglect on a letter cancellation task or on sensory testing. Investigations revealed an ischaemic stroke with haemorrhagic transformation in the right occipital and mesial temporal lobe. On magnetic resonance angiography he had a chronic left carotid occlusion, a mild right carotid stenosis, and bilateral vertebral artery stenoses. Since then, he has suffered from severe topographical disorientation, primarily in new environments.

$\mathrm{CO}$ reports that he can recognise buildings that were familiar to him before the stroke. He can also form mental images of well-known landmarks such as the Empire State Building and the Boston Public Gardens, and of the house he lived in as a child. When asked to imagine standing in front of the Massachusetts State House (a location well known to him from years of living in Boston), he accurately described the surrounding buildings and their locations. However, he reports that when he looks at a place that he's encountered post-stroke, all he gets is a feeling of familiarity that does not allow him to determine where the place is or when he saw it. He also reports an inability to understand how various landmarks relate to each other in large-scale space. Despite this difficulty, he can follow a map and frequently rides the Boston subway alone.
Unlike GR, CO was able to learn a certain amount of topographical information. For example, he soon learned the location of the rooms in the lab and would travel between them unassisted. After his first visit to the lab he needed to be guided all the way back to the subway station, but after several visits he could find his way out of the building and to the subway on his own as long as he was first guided to the elevators. (It should be noted, however, that the building is on the same block as the subway station. Furthermore, he still needed to be directed to the elevators even though our building has a relatively simple floor plan.) Thus, the amount of topographical information he can learn is limited but not zero.

CO's language and reasoning skills appear to be normal, and his conversation is appropriate and varied. In addition to his topographical difficulties, he also has limitations of his peripheral vision on the left side.

\section{Navigational abilities}

In addition to the experiments reported in the Experimental Investigations section of this paper, we also performed a number of ad hoc tests of GR and CO's navigational abilities. Although these tests were informal, we describe them here to provide a general sketch of the nature of GR and CO's impairments.

\section{Real-world topographical learning}

Over the course of several days, we took GR and his wife on three walks around the MIT campus, which neither had visited previously. Each walk followed exactly the same path. At several places in the walk, we would stop and point out different buildings and landmarks, tell them something about each one and ask them to remember their appearance for later testing. Immediately after the completion of the third walk, both GR and his wife separately performed a forced-choice recognition test in which they saw photographs of 14 landmarks (mostly buildings) from the walk and 14 novel landmarks (from another part of the MIT campus that they had not visited) and were required to report whether they had seen each one or not. GR's 
performance was $7 / 14$ correct hits for the familiar landmarks and 13/14 correct rejections for the unfamiliar landmarks. In contrast, his wife obtained a perfect score on this test. Given the extensive nature of the training regime, GR's score strongly suggests an impairment in learning the appearance of new real-world places. Interestingly, several of the seven familiar landmarks that he got right had an obvious distinctive feature that had been explicitly pointed out to him. For example, one building had a distinctive clock tower, and another one was an unusual triangular shape. Thus, GR was occasionally able to use distinct visual features to identify places even though he was unable to recognise them holistically - a common pattern among topographical disorientation patients.

GR did not appear to have any obvious problems in understanding the spatial structure of his immediate environment. When given a floor plan of the building at MIT in which he was being tested, he was able to successfully follow a route marked on the plan. However, when the plan was taken away, he had great difficulty finding his way back to the lab even though he had more than 30 min experience with the environment and his starting position was less than 30 feet from the goal (Figure 2). In fact, the route he took resembled a random walk. At one point, he walked right past his destination without recognising it, and only knew for sure that he was in the right place when he identified his wife in the room. Thus, his on-line appreciation of the layout of immediately visible space appears to be intact, but he cannot find his own way without assistance.

We were unable to perform the MIT landmark recognition test on CO because he suffered from a medical problem that made it difficult for him to walk around campus. In lieu of this, we tried to see whether he could learn a simple video game environment consisting of three connected rooms. After about $30 \mathrm{~min}$ experience navigating through this environment (via button presses) he was asked to sketch a map of it. He declined to do so, stating that it was beyond his abilities. When asked how many rooms there were, he replied that he did not know, but that there were at least 10 . Given the simplicity of this artificial environment, CO's complete inability to learn it is striking.

\section{Map drawing}

After several days of testing, we asked both GR and his wife to sit at different tables and independently sketch (1) the shape of Florida, (2) a bicycle, (3) a floor plan of their hotel room in Boston, (4) a floor plan of the first floor of the house they had been living in for the last 8 months (all post-stroke), (5) a floor plan of the main room of the lab, and (6) a floor plan of the house they lived in 10 years ago (pre-stroke). GR drew the map of Florida and the bicycle perfectly. His plan of the hotel room was notably different from the one drawn by his wifefor example, he reported that there was only one bed when in fact there were two, and he placed the bed on the wrong side of the room (Figure 1a). His plan of the first floor of his current house was somewhat better but was not very detailed and included some major errors. For example, he wrongly reported that there was no bathroom on this floor (Figure 1b). He was unable to produce any floor plan of the main room of the lab, leaving this page blank. When his wife reminded him that this was the room in which he had just spent an hour reading the newspaper and in which we had all had breakfast in that morning, he replied that he didn't know what room she was talking about (although he appeared to remember these events). In contrast to this near total inability to produce accurate maps of new environments, GR produced an accurate map of the house he lived in prior to his injury (Figure 1c). He reported that his memory of this house was "all there" and he could imagine walking around in it. When asked to produce a perspective of a room when he was in it, he did so with ease, and he was also able to create an accurate floor plan of the room.

In contrast, $\mathrm{CO}$ found it very difficult to produce a floor plan of the lab both when he was in it and when he was taken to another room and asked to recall it. However, although the maps he produced were quite impoverished, they were not entirely inaccurate, demonstrating some ability to encode topographical information. This contrasts with GR's absolute inability to recall anything 
EPSTEIN ET AL.
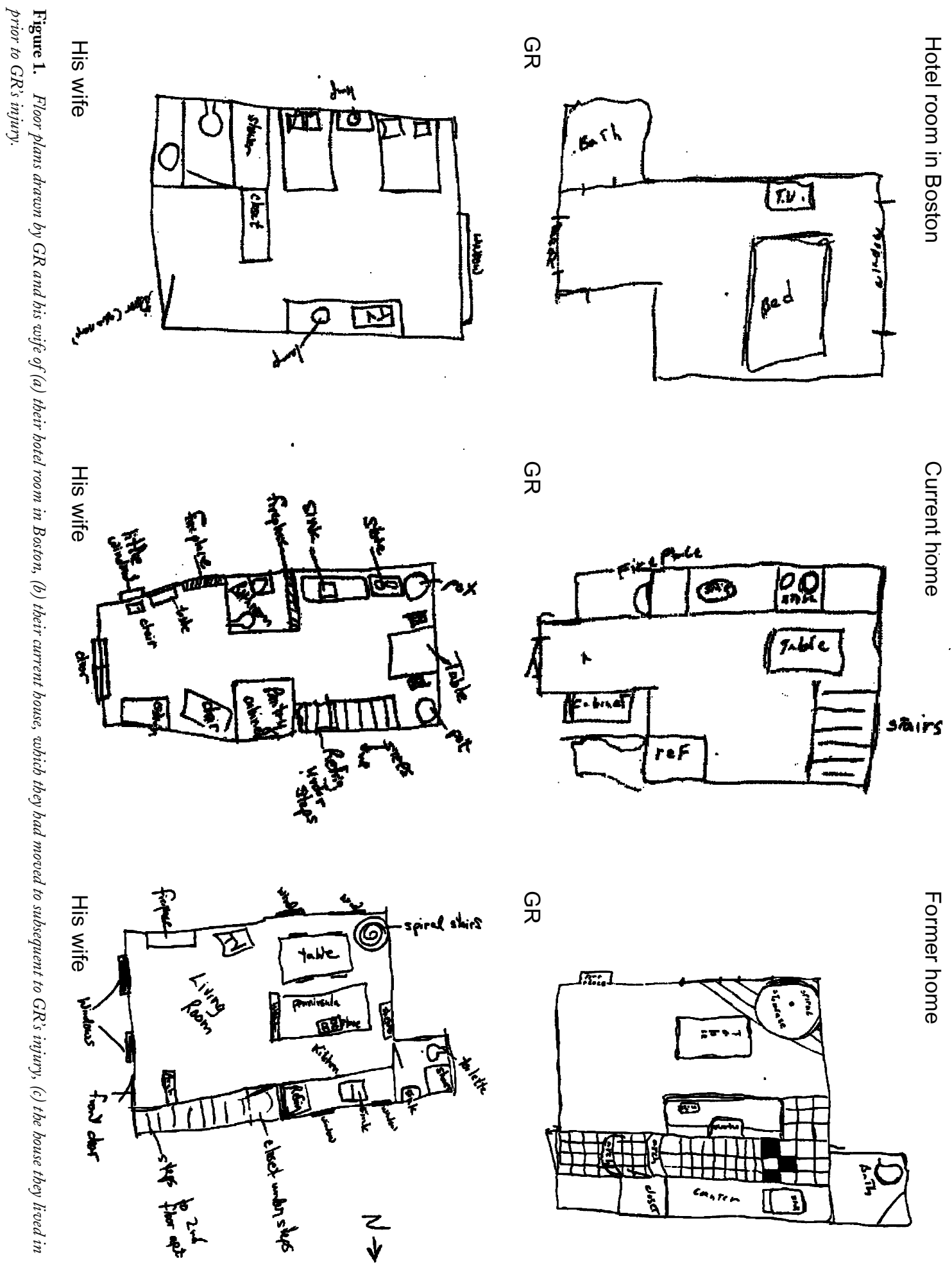


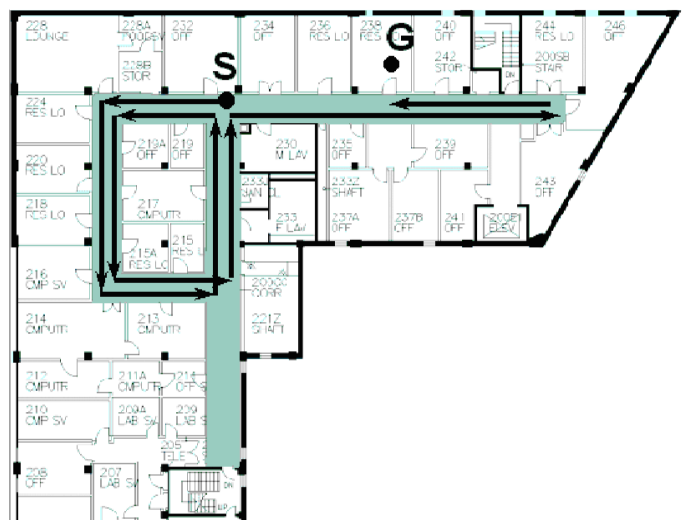

Figure 2. Floor plan of the building at MIT in which GR was tested. Corridors are highlighted in grey. GR was asked to find the main room of the lab (marked $G$ on the map). Starting location (marked S) was a point approximately 30 feet down the hall. GR's route is marked by arrows. GR found this task very difficult, and only knew for certain that he had reached his goal when he caught sight of his wife through the glass door of the lab. In contrast, he was able to follow a route marked on a plan of the same floor without any problem or hesitation.

about the main room of the lab once he had left it. $\mathrm{CO}$ was also able to draw a detailed floor plan of the apartment he was living in at the time (and had lived in for 20 years). Several months after he had moved from this apartment, we asked him to draw such a floor plan again, and he produced one that was substantially the same as the first (Figure 3). Thus, their map drawing performance indicates that GR and $\mathrm{CO}$ are primarily impaired at sketching places experienced after their strokes, but are unimpaired at sketching places experienced before their strokes. This pattern is indicative of deficits in encoding but not recall.

\section{Scene perception}

As noted earlier, GR can navigate with the aid of a floor plan, suggesting that his perceptual abilities are largely intact. Interestingly, however, he reports that he does have a perceptual deficit, which he describes as an inability to understand scenes when they are too complex. For example, he reports that he often has trouble using maps because they usually are too busy with details. It is unclear whether this apparent perceptual problem is a result of his field cut, some other separate perceptual problem, or a memory problem (see discussion). $\mathrm{CO}$ did not complain of a similar deficit. GR was an accomplished artist prior to his injury, producing paintings of striking complexity. Tragically, he reports that he cannot now appreciate his own paintings.

\section{Other cognitive abilities}

\section{Face recognition}

GR also reported some difficulties with face recognition. On his second visit, we examined his ability to learn new faces by having him perform a forcedchoice recognition test on seven familiar and seven unfamiliar faces. The familiar faces were personnel from the lab with whom he had at that point had a good deal of experience, spanning at least 3 days. All the people in the photographs had a towel wrapped around their head in order to minimise the use of hair as a nonfacial cue. Despite the difficulty of this task, he correctly identified 6/7 familiar faces, and made no false alarms. Surprisingly, the one face that he did miss was the first author of this paper, with whom he at that point had had considerable experience. GR also performed normally on a difficult famous face recognition test (see Experiment 4, following) and on the Benton Face Recognition test (see following). Thus, he has some ability to recognise faces. Consistent with this, GR reports that he can remember a small number of faces, but that he quickly "loses track" if he has to remember too many.

$\mathrm{CO}$ also appears to suffer from some prosopagnosia, as evidenced by his low performance on the Benton Face Recognition test (see following; see also Experiment 4). Interestingly, he does not complain of face recognition difficulties, but rather attributes his inability to recognise people to a lack of interest in their appearance.

\section{Episodic memory}

At first glance, GR's episodic memory appeared to be largely intact. As mentioned, he could recall the events of the previous day accurately. Furthermore, he reports that he can follow the plot of a movie without any difficulty. However, both he and his wife report that he does have some problems with 


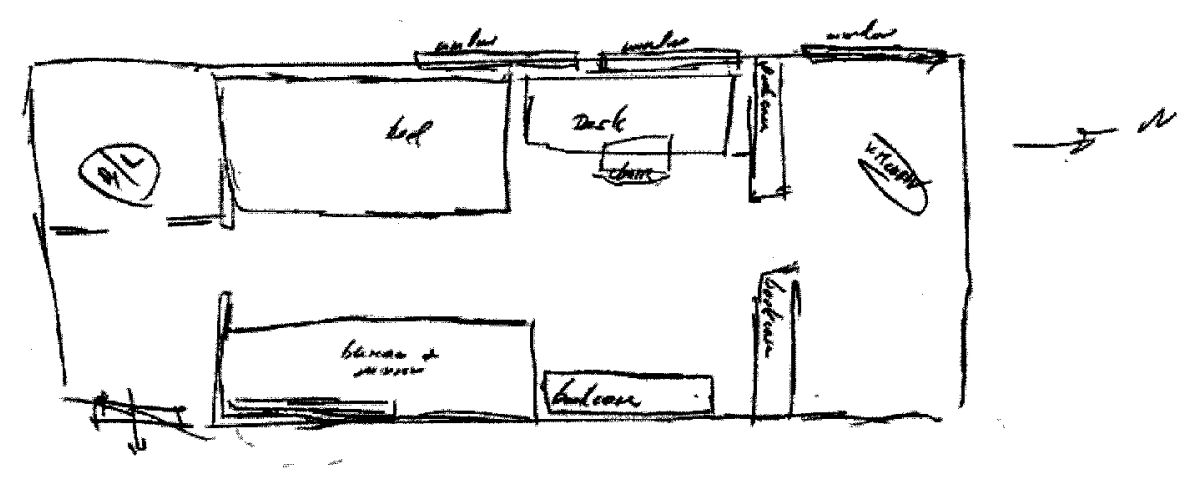

$\mathrm{CO}$, (former) home, June 99

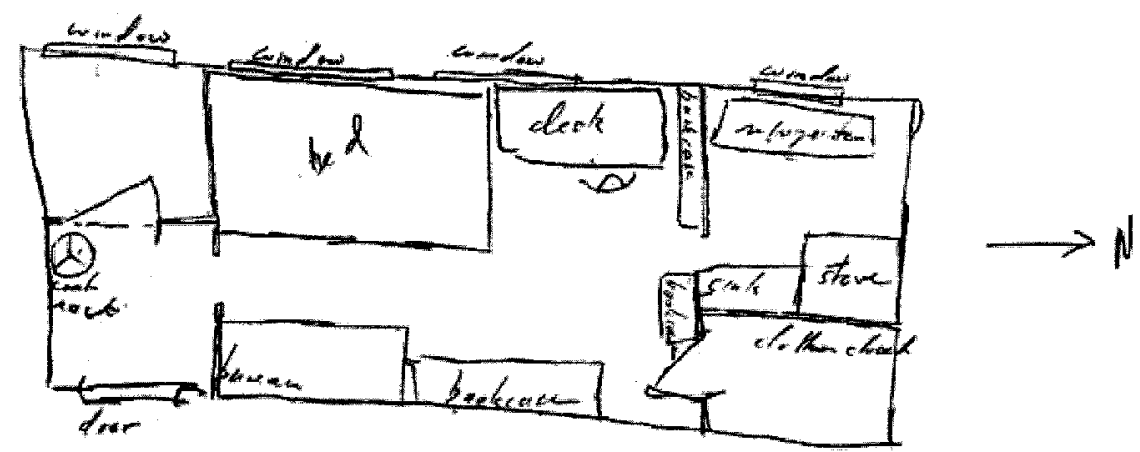

Figure 3. Two floor plans drawn by $\mathrm{CO}$ of the apartment he lived in for over 20 years. The first plan was drawn when $\mathrm{CO}$ still lived in the apartment; the second was drawn several months later when he had moved out. Note that the two plans correspond very well. CO reported that it was quite easy to produce these floor plans.

episodic memory. For example, he stated that he remembered very little about a trip to Italy they had taken several months previously. His wife reports that he has great difficulty keeping track of time: For example, before their trip to Boston, he repeatedly asked her what day they were supposed to leave and seemed unable to encode this information. We observed a similar phenomenon during testing: During the tests that required repetition of the same procedure several times, he sometimes seemed unable to clearly remember how many times he had repeated the procedure. Thus, he does appear to have a subtle episodic memory deficit, which may manifest itself as an inability to separately remember successive events that are not easily distinguishable from each other.

$\mathrm{CO}$ also reports some problems with his episodic memory. He relies on lists to remember appointments, stating "if I didn't have a list, I'd probably forget why I walked in the door here." Despite this claim, he has never missed an appointment, nor is he ever confused about the purpose of the testing appointments. He can accurately report the day of the week. He remembers the experimenters clearly from appointment to appointment, as well as the details of the various experiments 
when they are repeated across appointments. When queried about his episodic memory difficulties, $\mathrm{CO}$ reported that he will usually remember an event if he is reminded of it, but that he has difficulty spontaneously regenerating events. Interestingly, he reports that even when he is reminded of an event and remembers it, he cannot remember "where it was or when it was." When he reads, he finds it difficult to follow a story if it skips around. However, when queried, he was able to accurately report what he had read in the newspaper the previous day.

\section{Formal neuropsychological tests}

Formal neuropsychological testing was performed on GR in September 1998 and on CO in December 1998. Test performances were compared to agematched peers ${ }^{3}$.

GR exhibited mild to moderate impairment in memory relative to what would be predicted for his intellect (WAIS-R Full Scale IQ = 122; 93rd percentile; superior range) and level of education. These memory problems were more apparent on visual than on verbal memory tests. On a test of memory for conceptually organised text passages (Wechsler Memory Scale Logical Memory [WMS-III]; Wechsler, 1997b) his performance was average for both immediate (LM I $=25$ th percentile; standard score SS $=90$ ) and delayed recall (LM II $=50$ th percentile, $\mathrm{SS}=100)$. In contrast, his score on the WMS-III Face Memory subtest was in the low average range for both immediate (9th percentile; $\mathrm{SS}=80$ ) and delayed recall (16th percentile; $\mathrm{SS}=85$ ). On a test of memory for scenes (WMS-III Family Pictures subtest), immediate recall was deficient (2nd percentile; SS $=69$ ) and delayed recall was borderline impaired (5th percentile; SS $=76$ ). GR also had difficulty learning word lists: on the Rey Auditory Verbal Learning Test (Spreen \& Strauss, 1998) his immediate recall was average (63rd percentile; SS $=105$ ) but after the interference list his performance fell to the low average range for both immediate (14th percentile; SS =84) and delayed recall (16th percentile; $\mathrm{SS}=85$ ). On a recognition test of the items of the list, he successfully recognised all 15 , but made 8 false alarms. Performance on the 7/24 Spatial Recall Test (Rao, Hammeke, McQuillen, Khatri, \& Lloyd, 1984), which required him to remember seven locations on a $6 \times 4$ checkerboard, was 66 th percentile $(S S=106)$ for immediate and 2nd percentile (SS = 69) for delayed recall. Performance on the Benton Face Recognition test was normal (42nd percentile). Performance on the Boston Naming Test was at ceiling, indicating that language abilities were intact. Thus, GR exhibits some impairment on tasks that require delayed recall of visual information (WMS-III Face Memory subtest) or verbal information that is not organised in terms of a story or plot (Rey AVLT), and is clearly impaired on tasks that required delayed recall of spatial information (7/24 Spatial Recall Test) or stimulus location (WMS-III Family Pictures subtest). In contrast, his ability to recall organised verbal information (WMS-III Logical Memory subtest) appears to be intact.

As with GR, CO's memory performance was generally below what would be expected based on intellect (WAIS-III Full Scale IQ = 134; 99th percentile; very superior range) and education level. Also like GR, there was some evidence that visual memory problems were more pronounced than verbal memory problems. Memory for text passages (WMS-R Logical Memory) was average for both immediate (27th percentile; SS =91) and delayed recall (48th percentile; $S S=99$ ). In contrast, performance on the WMS-III Faces subtest was low average for both immediate (16th percentile; SS = 85 ) and delayed recall (9th percentile; $\mathrm{SS}=80$ ), and performance on the Family Pictures subtest was deficient $(<1$ st percentile; SS < 62) for both immediate and delayed recall. On the Warrington Recognition Memory Tests (RMT), he scored in the average range for words (50th percentile; SS $=100$ ) and scenes (Warrington Topographical Recognition Memory Test; 29th percentile; SS =92), and in

\footnotetext{
${ }^{3}$ The formal neuropsychological testing reported in this section was performed prior to the beginning of our combined investigation of GR and CO (i.e., prior to the main experimental investigations of this paper). Consequently, GR and CO were not tested on exactly the same battery of tests.
} 
the deficient range $(<5$ th percentile; SS $<75)$ for faces. The low score for faces on this test as well as the WMS-III may reflect prosopagnosia rather than a memory problem, as he scored in the deficient range $(<1$ st percentile; SS $<62)$ on the Benton Face Recognition test. Verbal list learning (Rey AVLT) was average on immediate recall (45th percentile; SS $=98$ ), but after hearing an interference list performance fell to the deficient level (0/15 words recalled) for both immediate and delayed recall. On a recognition test for these words, he recognised $11 / 15$ and made one false alarm (39th percentile, $S S=96$ ). There was a large discrepancy on tests of memory span, which may indicate a decline in spatial memory: digit span was superior (9+ forward and 7 backwards; both 99th percentile) but spatial span was only average ( 5 forward and 5 backward; 37th and 50th percentiles respectively). Language abilities were intact (Boston Naming Test performance was at ceiling). Thus, like GR, $\mathrm{CO}$ shows some impairment at recall of visual information (WMS-III Face Memory subtest) and verbal information that is not organised into a narrative (Rey AVLT), and clear impairment at tasks that require recall of stimulus location (Family Pictures subtests of WMS-III). In contrast, his ability to recall organised verbal information (WMS-R Logical Memory subtest) is more preserved.

\section{MRI RESULTS}

In order to determine the extent of their lesions, structural MRI images were taken of both subjects. Delineation of the lesions was based either on spoiled GRASS coronal images at $1.1 \mathrm{~mm}$ thickness (GR) or anatomical T-1 weighted MPRAGE MRI sequences yielding $1.25 \mathrm{~mm}$ thick coronal images (CO). The anterior-posterior extent of the lesions was verified on axial images.

GR has bilateral occipital-temporal lesions (Figure 4). In the left hemisphere, the posterior parahippocampal gyrus (including parahippocampal cortex) is severely damaged, with only minimal injury to the most caudal aspect of the hippocampus. There is also moderate damage to the medial fusiform and inferior lingual gyri. In the right hemisphere, the hippocampus is spared, but there is damage to the posterior end of the parahippocampal gyrus near the parahippocampallingual boundary. More posteriorly, the lesioned area extends along the collateral sulcus to include significant portions of the inferior lingual gyrus and the medial fusiform gyrus, as well as most of the medial occipital lobe below the calcarine fissure.

$\mathrm{CO}$ has a right-hemisphere occipital-temporal lesion. Right posterior hippocampus and parahippocampal gyrus are severely affected. More posteriorly, the damage covers large portions of the inferior lingual and medial fusiform gyri, as well as substantial portions of the medial occipital lobe including most of right calcarine cortex. In addition, diffuse foci of increased signal on $\mathrm{T}-2$ weighted images was present in periventricular white matter in the corona radiata and centrum semiovale with a lacunar infarct of the right putamen. There was also moderate degree of diffuse involutional changes with increased ventricular size, particularly notable in the right temporal horn with ex vacuo dilitation.

The structural MRIs suggested that the PPA was severely if not completely damaged in both hemispheres for GR, and in the right hemisphere for CO. In order to confirm this for GR, we used a high-field 3-T magnet to perform a functional scan in which he viewed scenes, faces, and objects in the same blocked fMRI design that we have used to localise the PPA in all our previous experiments (see Epstein et al., 1999, for details; Epstein \& Kanwisher, 1998). A Kolomogorov-Smirnov test found novoxels in the parahippocampal region that fit our previously established criterion for inclusion in the PPA (i.e., greater response to scenes than to faces and objects at a uncorrected significance level of $p<.0001)$. This result contrasts sharply with the pattern seen in over $95 \%$ of (mostly young or middle-aged) normal subjects, in whom a large activated region can be identified in both left and right parahippocampal cortex using this comparison (see Figure 5). A similar test was used to identify voxels that responded significantly more to faces than to objects. This comparison revealed a region of faceselective voxels in the right fusiform gyrus consistent with the location of the Fusiform Face Area 


\section{GR}
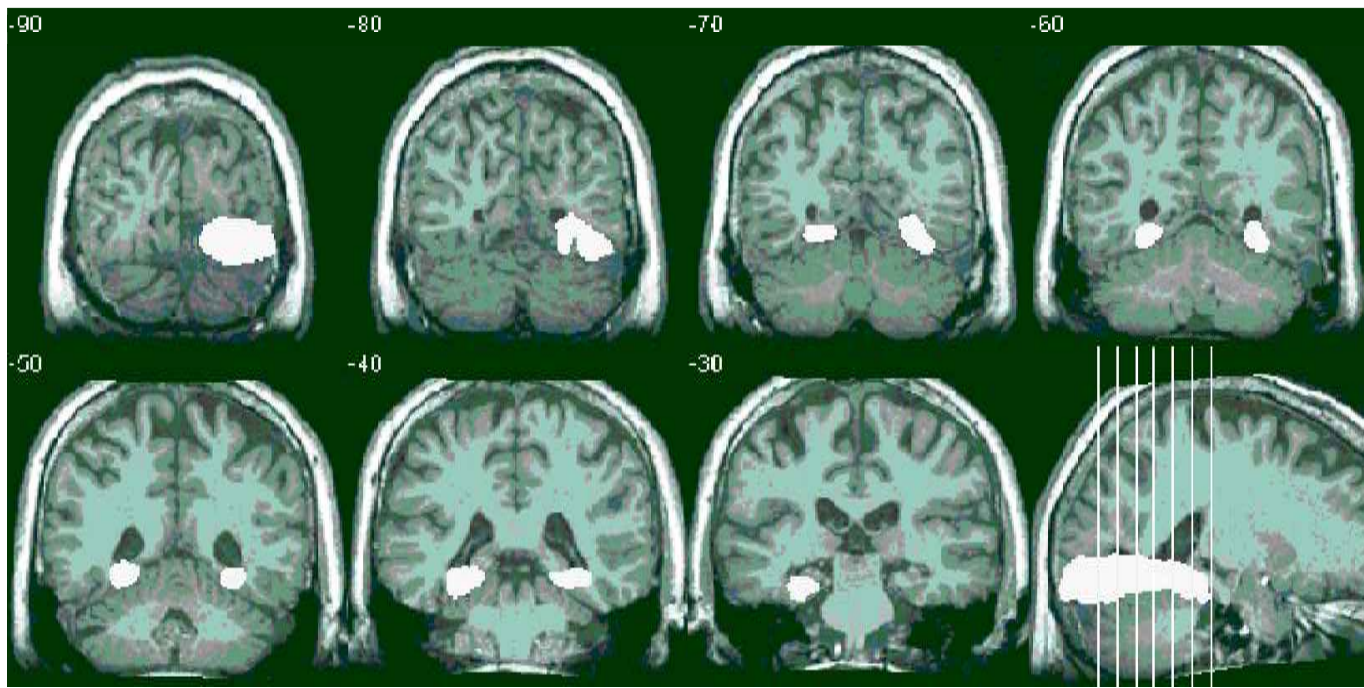

$-40$

$-30$
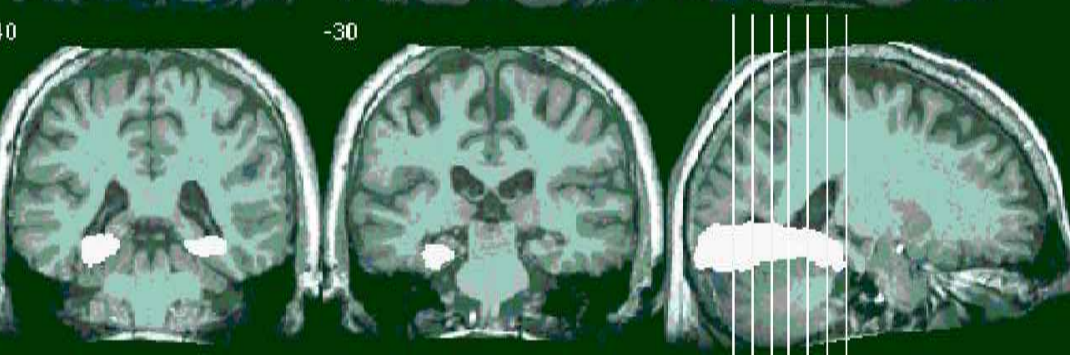

\section{$\mathrm{CO}$}

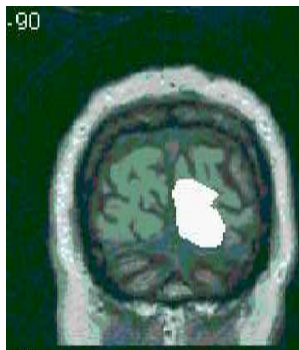

$-80$

$-70$
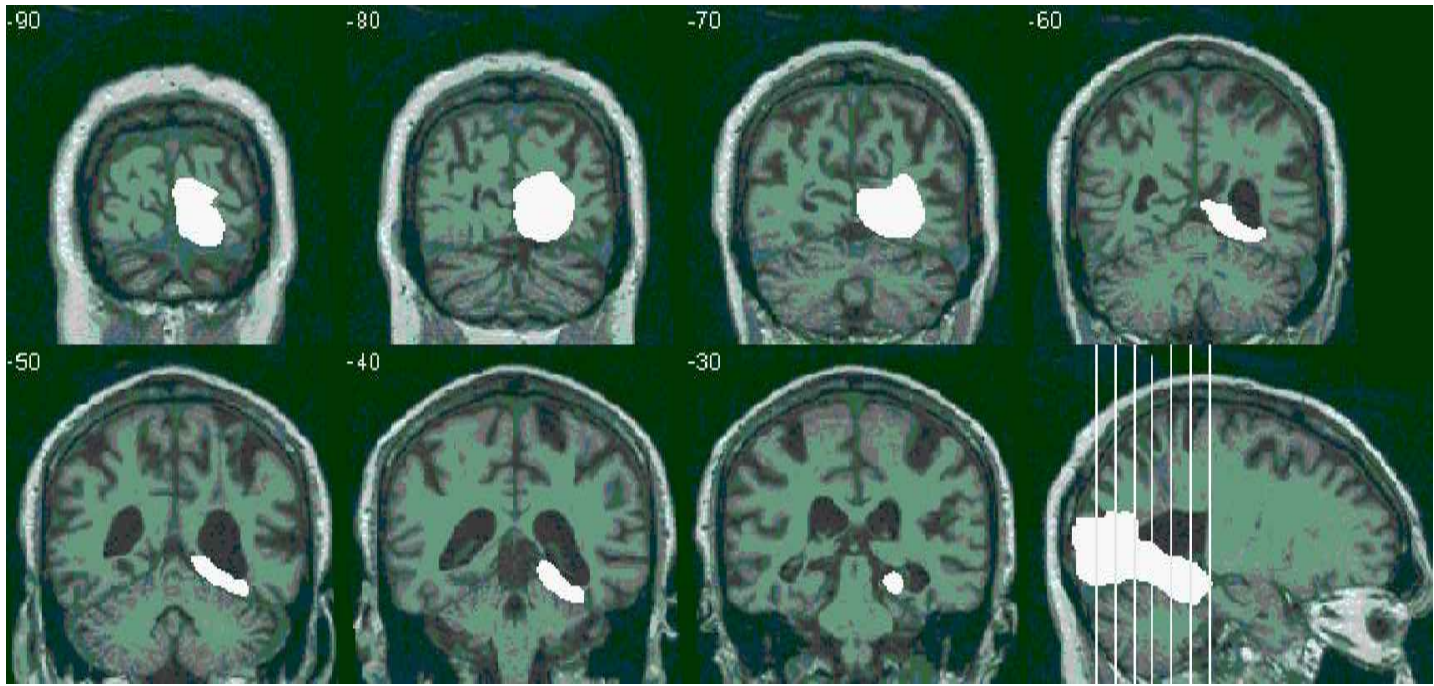

Figure 4. Structural MRIs of GR and CO showing the extent of the damaged region (marked as white). Images have been normalised using SPM99 and a cost function masking algorithm (provided by Matthew Brett) into standard stereotactic space (Talairach E Tournoux, 1988) using the Montreal Neurological Institute template (Evans et al., 1993). Numbers indicate y-Talairach coordinate for each coronal slice; corresponding slice planes are also indicated on the sagittal image. 


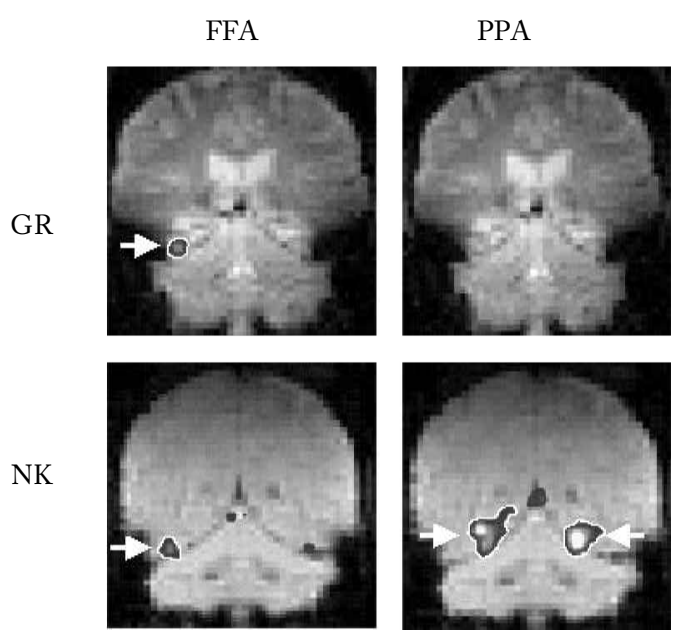

Figure 5. Top row shows a single slice from a functional MRI scan performed on GR. Bottom row shows results from a normal subject (NK) for comparison. Voxels that responded more to faces than objects (left column) or more to scenes than faces and objects (right column) in a Kolomogorov-Smirnov test are highlighted by white arrows. GR has a functioning face area (FFA), but there is no evidence of a place area (PPA).

(FFA) commonly seen in normal subjects (Kanwisher, McDermott, \& Chun, 1997). Thus, the MRI was sensitive enough to detect functional differences: an FFA was found, but no PPA. When combined with the anatomical results, this strongly suggests that GR has no PPA.

Because of safety concerns (presence of a femoral artery aneurysm clip), we did not perform a similar high-field functional MRI of CO. However, as Figure 4 demonstrates, $\mathrm{CO}$ clearly has damage to the parahippocampal-lingual region in the right hemisphere.

\section{EXPERIMENTAL INVESTIGATIONS}

In this section, we report the results of four experiments designed to probe the consequences of damage to the lingual-parahippocampal region in general and the PPA in particular. Our primary concern in these experiments was to test GR and CO's ability to perceive, remember, and recognise different kinds of visual material. Based on our previous $\mathrm{fMRI}$ work, we predicted that $\mathrm{GR}$ and $\mathrm{CO}$ would have more difficulty with scene-like stimuli (i.e., stimuli that convey information about the geometry of a space one can navigate through or act within) than with object-like stimuli. Such a deficit could account for at least some of the problems GR and $\mathrm{CO}$ encounter in real-world navigation.

Beyond determining the kind of visual material that gets processed in parahippocampal cortex, we also wanted to determine the nature of the processing that takes place on that material. In particular, we wanted to determine if parahippocampal cortex is involved in perceptual encoding, memory encoding, or recognition. As discussed earlier (Introduction), our fMRI results provided some evidence against place recognition as the sole function of the PPA, but did not allow us to distinguish between perceptual and mnemonic encoding. Experiments 1 and 2 examine the role of parahippocampal cortex in perceptual encoding, Experiment 3 examines its role in mnemonic encoding, and Experiment 4 examines its role in place recognition.

\section{Experiment 1: Two-dot task}

The first hypothesis we examined was that parahippocampal cortex plays a role in perceiving the shape of the local environment. In our previous fMRI work, we observed that the PPA responds much more strongly during scene viewing than during object or face viewing, even when subjects do nothing more than watch the scenes passively without performing any other task. This suggested that scene perception alone may be sufficient to activate the PPA. Previous reports have indicated that damage to parahippocampal cortex and other medial temporal regions leads to problems with memory rather than perception (Bohbot et al., 1998; Maguire, Burke, Philips, \& Staunton, 1996; Pigott $\&$ Milner, 1993). However, all of these previous results were obtained on patients who had unilateral lesions. GR has a bilateral occipital-temporal lesion, including clear damage to parahippocampal cortex in both hemispheres; furthermore, he reports some kind of perceptual deficit. We hypothesised that this deficit might involve difficulty perceiving or interpreting the geometry of scenes and other stimuli with a similar geometric structure. 
In this experiment, subjects viewed either object shapes or spatial layouts. Stimuli consisted of black-and-white photographs of real-world stimuli (empty rooms vs. common objects) or abstract stimuli made out of Lego blocks (Lego "scenes" vs. Lego "objects"). These stimuli have been previously shown to differentially activate the PPA in normal subjects (Epstein et al., 1999; Epstein \& Kanwisher, 1998). Two red dots were superimposed on each photograph (Figure 6). The task was to report which of the two red dots was over the closer part of the object or scene. This task was designed to have no memory component (apart from remembering the instructions), but to require accurate 3D perception of objects and scenes.

If $\mathrm{GR}$ or $\mathrm{CO}$ have difficulty perceiving or interpreting the spatial layout of scenes, then we predicted they would do worse when the stimuli are empty rooms or Lego scenes than when they are real-world or Lego objects. Note that the critical comparison is between the Lego scenes and the Lego objects, as these stimuli are made of the same materials and differ primarily in their surface geometry.
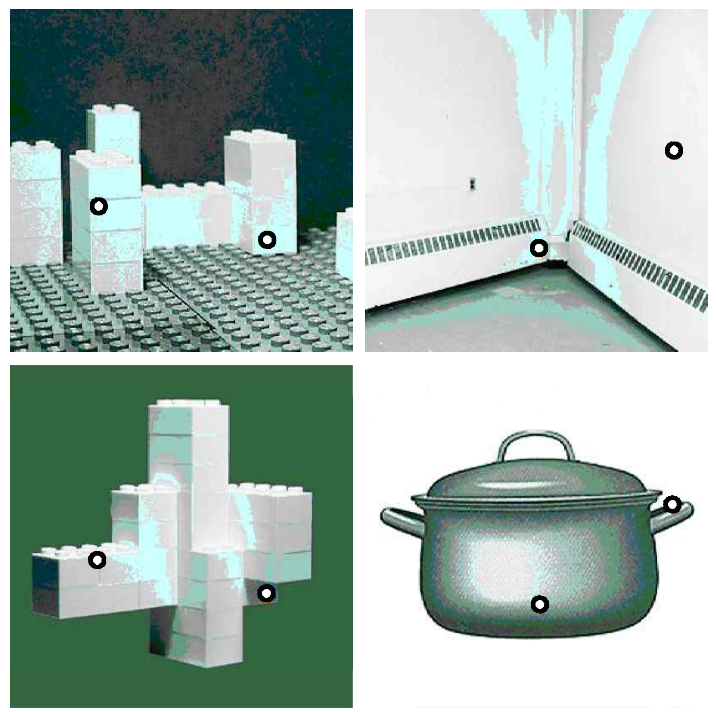

Figure 6. The four kinds of stimuli used in Experiment 1. Top left: lego layouts, top right: real-world layouts (empty rooms), bottom left: lego objects, bottom right: real-world objects.

\section{Method}

Testing for all experiments was done on a Macintosh computer. The experiment consisted of 80 trials (20 of each stimulus type, randomly intermixed). Subjects began each trial by pressing the space bar. A black-and-white photograph from one of the four stimulus categories (see earlier) then appeared on the screen, with two red dots on it. The task was to determine which of the two red dots indicated the closer part of the scene or object. Subjects pressed the "f" key if the leftmost red dot was closest, and the " $j$ " key if the rightmost dot was closest. They were instructed to respond as quickly as possible without making errors. Stimuli remained on the screen until a response was made.

The stimulus set was constructed so that each empty room was matched to a common object that had red dots in the exact same location, and each Lego layout was similarly matched to a Lego object. Thus, the locations of the red dots were counterbalanced across scene and object stimuli, controlling for any effects of visual field cuts.

\section{Results and discussion}

Results for GR and $\mathrm{CO}$ and four elderly normal subjects are shown in Table 3. Both GR and CO could do this task without difficulty, and their performance was near ceiling. There was no evidence that they had any more trouble with the empty rooms or Lego scenes than with the real or Lego objects. Reaction times were slightly longer for GR and $\mathrm{CO}$ than for normal subjects, as one would expect given their visual field deficits; however, this

Table 3. Experiment 1 (two-dot) results (SD in parenthesis)

\begin{tabular}{llll}
\hline & $G R$ & $C O$ & Normals \\
\hline Proportion correct: & & & \\
Object & 0.95 & 0.80 & 0.93 \\
Lego object & 1.00 & 0.95 & 0.98 \\
Empty room & 1.00 & 1.00 & 0.99 \\
Lego room & 0.95 & 1.00 & 0.96 \\
& & & \\
Mean RT: & & & \\
Object & 2435 & 2932 & $2161(980)$ \\
Lego object & 2052 & 3245 & $2029(921)$ \\
Empty room & 2744 & 2291 & $1788(959)$ \\
Lego room & 1904 & 2281 & $1630(1127)$ \\
\hline
\end{tabular}


difference did not appear to be significant. Importantly, there was little evidence that GR or CO took longer to make their perceptual judgements for the real or Lego scenes than for the real or Lego objects. Thus, contrary to our initial hypothesis, this experiment found no clear evidence for selective impairment in the perception of scene-like stimuli.

\section{Experiment 2: Different-views matching task}

The previous experiment demonstrated that GR and $\mathrm{CO}$ can extract information about the perceived distances between themselves and the surfaces of an object or scene. Thus, in at least one sense, their perception of these surfaces is preserved. However, this does not mean that their ability to represent the layout of a scene is unimpaired. The two-dot task requires on-line comparison of two surfaces in egocentric coordinates. GR and CO would be able to perform this task even if they could not (1) represent more than two surfaces at a time, (2) represent surfaces in other than strictly egocentric coordinates, or (3) sustain surface representations for longer than the time it takes to make a comparison. It has been suggested that the parietal lobes are involved in on-line processing of surface geometry in egocentric coordinates (Milner \& Goodale, 1995); thus it is possible that the parietal lobes (which are intact in GR and CO) may be sufficient to perform the two-dot task even in the absence of more durable or complex surface representations in parahippocampal cortex.

In Experiment 2, we examine GR and CO's ability to create and sustain a representation of the geometry of an object or scene-like layout. The task was to view a photograph of a Lego object or Lego scene, hold it in memory for $10 \mathrm{~s}$, and then determine whether a second object or scene was the same as the first. In "same" trials the second photograph depicted the same scene or object depicted from a different viewing angle. In "different" trials the second photograph depicted a different scene or object chosen to be similar to the first one (Figure 7). Thus, in order to successfully perform this task, subjects had to form a representation of the surfaces of the object or scene, sustain this representation in memory for $10 \mathrm{~s}$, and then align it (presumably using mental rotation), to the second object or scene.

\section{Method}

The experiment consisted of two parts, each with 40 trials. In the first part, the stimuli were black and white photographs of objects made out of Lego blocks. In the second part, the stimuli were photographs of "scenes" made out of Lego blocks, which were designed to have a geometric structure similar to real-world places such as a room or a city street.

Subjects began each trial by pressing the space bar. A photograph of a Lego object or scene appeared on the screen for $3 \mathrm{~s}$, followed by a 10-s blank interval. A second photograph then appeared depicting either (1) the same object or scene as the first photograph shown from a different viewing angle (offset approximately 35 degrees), or (2) a different object or scene. Subjects were instructed to press the " $\mathrm{j}$ " key if the two photographs depicted the same object or scene and the " $\mathrm{f}$ " key if they did not. They were told to take as much time as they needed to make a decision but no extra time. The second photograph remained on the screen until a response was made. Half the trials were "same" trials and half were "different" trials.

\section{Results and discussion}

Figure 8 shows results for GR and $\mathrm{CO}$ as well as for six elderly normal controls. GR's and CO's performance was comparable to that of the normal controls. Furthermore, GR and $\mathrm{CO}$ performed just as well when the stimuli were Lego scenes as when they were Lego objects. These results are unlikely to reflect a ceiling effect as performance for both patients and controls was around 80\% correct. Thus, both patients appear to be able to form a representation of the geometry of both scenes and objects which they can sustain in short-term memory.

\section{Experiment 3: N-back recognition memory task}

The previous experiment failed to find any impairment in GR and CO's ability to form a representa- 
(a) Lego layout version

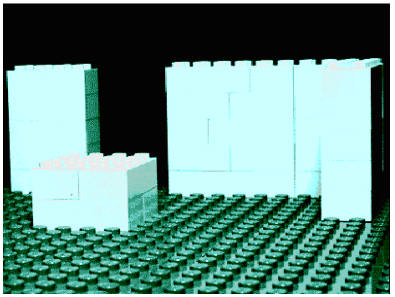

$3 \mathrm{~s}$

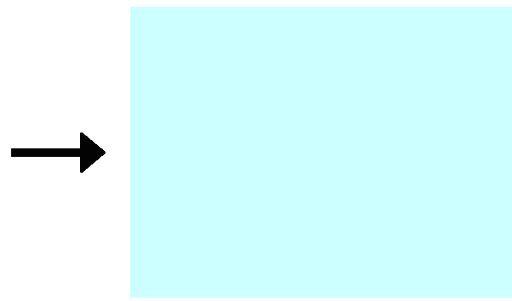

$10 \mathrm{~s}$

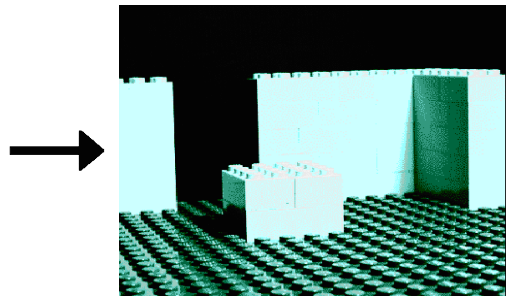

OR

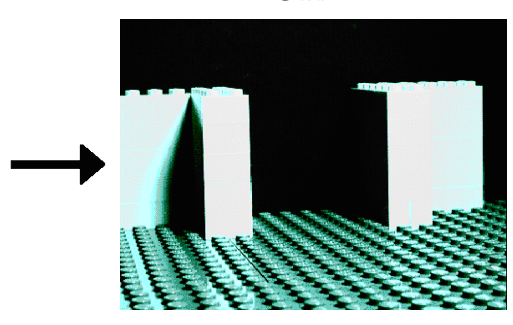

(b) Lego object version

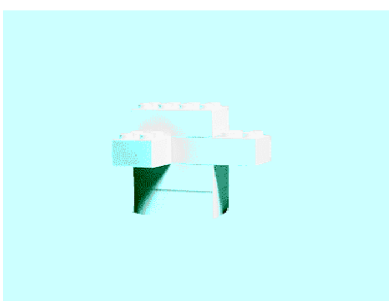

$3 \mathrm{~s}$

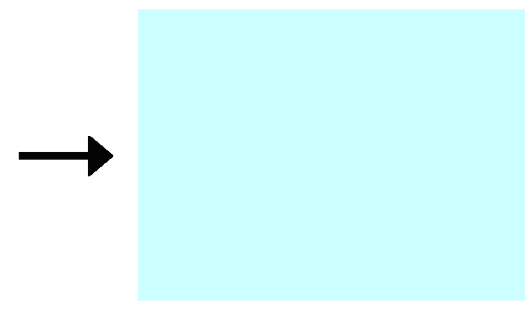

$10 \mathrm{~s}$

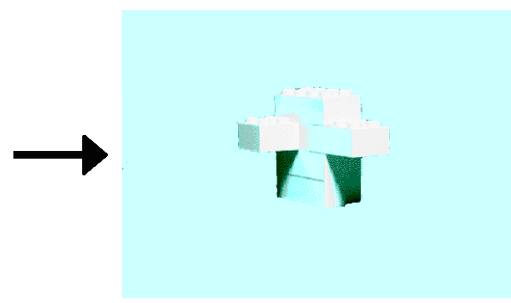

OR

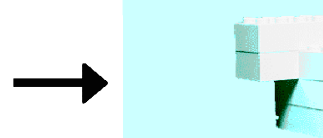

Figure 7. Stimuli and procedure for Experiment 2. (a) In the Lego layout version, subjects see a Lego layout for 3 s followed by a blank screen for $10 \mathrm{~s}$. A second layout then appears which is either the same layout as the first one but seen from a different angle, or a different layout. (b) The Lego object version is the same but with object stimuli.

tion of the geometry of a scene or object and maintain it for $10 \mathrm{~s}$ in the absence of the original stimulus. Not only does this result suggest that perception of scene-like stimuli is intact in these patients, it further suggests that some short-term memory functions are intact as well. However, we should note that subjects in this experiment were free to devote all their effort and attention in the 10second blank interval to maintaining a memory of the stimulus. Thus, it is possible that GR and CO can use rehearsal mechanisms to maintain the stimulus in short-term memory during the blank inter- 


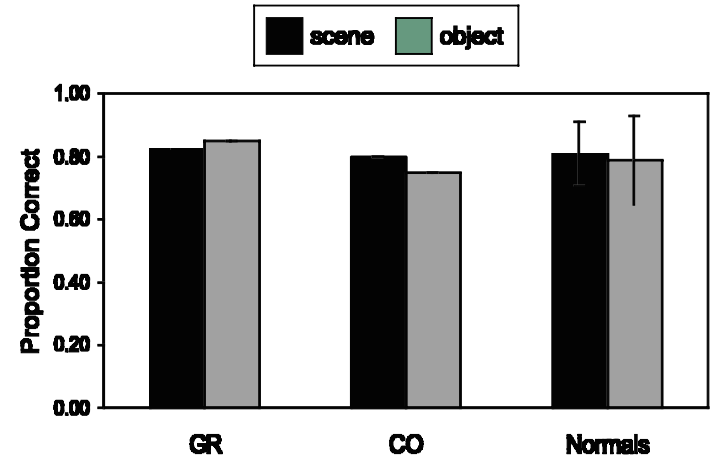

Figure 8. Results of Experiment 2. Performance over "same" trials and "different" trials is averaged together. Error bars show $1 S D$.

val, but are unable to form a more durable memory trace for the stimulus. This possibility was tested in the current experiment, which was designed to examine GR and CO's ability to learn new visual information.

We devised a continuous n-back recognition memory test in which subjects viewed a sequence of originally novel stimuli, some of which were repeated within the sequence at intervals of 1,3 , or 5 intervening images. For each stimulus, subjects reported whether they had seen it before in the experiment or not. In different runs in the experiment, the stimuli were black-and-white photographs of (1) Lego scenes, (2) Lego objects, (3) realworld landscapes, (4) novel objects made out of Sculpey clay, (5) unfamiliar houses, (6) unfamiliar faces, as well as (7) line drawings of nonsense objects, and (8) pronounceable pseudowords (Figure 9). Thus, we were able to test GR and CO's recognition memory for different kinds of materials at different time intervals in a paradigm in which the presence of intervening items between the two appearances of each stimulus made rehearsal difficult. Note that because none of the specific stimuli used in this experiment were familiar in advance, this task did not require an episodic memory component and could be done on the basis of pure visual familiarity with the particular visual stimulus.

Although many different stimulus classes were tested in this experiment, the critical comparison is between the Lego layouts and Lego objects, because these are best matched with regard to materials and differ primarily in their geometric structure (see Introduction). We predicted that if GR and CO showed a memory deficit for this task, it would be greater for the Lego layouts than for the
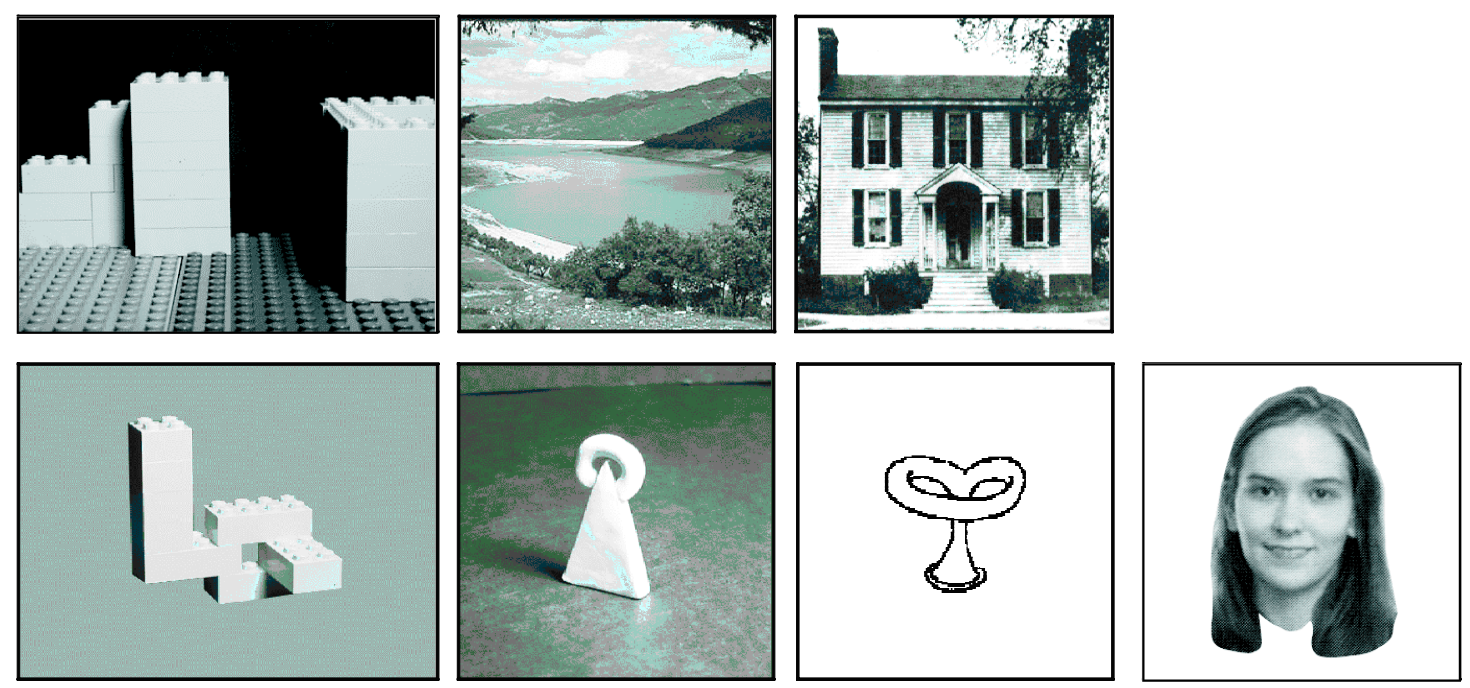

Figure 9. Stimuli for Experiment 3. Top row: Lego layouts, real-world scenes, houses. Bottom row: Lego objects, clay objects, nonsense objects, faces. In an eighth condition (not shown) subjects viewed pronounceable nonwords such as "wilch" or "thipper." 
Lego objects. In addition, a deficit for materials that convey information about the layout of surrounding space might also be apparent when GR and $\mathrm{CO}$ are tested on more realistic stimuli. If so, one would expect them to do poorly on the landscapes, but relatively well on the clay objects, line drawings of nonsense objects, faces, and pseudowords.

\section{Method}

For each subject, a complete experimental session consisted of being run once on each of the eight stimulus classes. Each run consisted of 90 trials; in each trial a stimulus was presented and subjects reported whether or not they had seen it before in the run (or indeed, ever) by pressing the " $j$ " key if they had or the " $f$ " key if they had not. Each stimulus was on the screen for either 1, 2, or $3 \mathrm{~s}$ (see following), and appeared promptly after the keypress report for the previous trial. Individual stimuli appeared either once (14 stimuli), twice (30 stimuli), or four times (4 stimuli) within a given run. Of the stimuli that were presented twice, 10 were repeated immediately (1-back), 10 were repeated with a lag of two intervening items (3-back), and 10 were repeated with a lag of four intervening items (5-back). In order to ensure that between-subject differences in performance were not due to stimulus differences, the same stimuli were assigned to the same conditions each time the experiment was run.

GR performed the experiment twice: once in August 1998, and again 9 months later. Because of a computer error, he was only tested on six of the eight categories (all except Lego layouts and Lego objects) at the later date. $\mathrm{CO}$ was also tested twice: once in February 1999, and again 6 months later. Reported results for GR and CO are the average of their two testing sessions. Normal subjects were only tested once. Stimulus presentation time was $1 \mathrm{~s}$ in GR's first testing session. In pilot testing, CO found this too fast for the critical categories of Lego layouts and Lego objects, so presentation time was extended to $2 \mathrm{~s}$ for these categories in his first testing session. All normal subjects were tested at these presentation rates ( $2 \mathrm{~s}$ for Lego layouts and objects, $1 \mathrm{~s}$ for other stimuli). In CO's second testing session, presentation time was lengthened to $3 \mathrm{~s}$ for all stimulus categories to ensure that any deficits found were memory deficits rather than problems with the nonmemory aspects of the task.

\section{Results and discussion}

Results from GR, CO, and 10 elderly normal controls are shown in Figure 10 and Table 4. Figure 10a shows results from the main comparison between Lego layouts and Lego objects. Both normal subjects and patients performed near ceiling for both Lego layouts and Lego objects at 1-back interstimulus intervals, verifying that GR and CO could successfully perceive the pictures as they were being presented. In contrast, there was a striking difference between the two patients and the normal subjects at 5-back intervals. Whereas normals did on average somewhat better on Lego layouts than Lego objects, both GR and CO showed the opposite pattern, doing much worse on Lego layouts than Lego objects. The 3-back condition showed a mixed pattern: Whereas GR did better on Lego objects than Lego layouts in this condition (similar to his performance at 5-back intervals), $\mathrm{CO}$ did better on Lego layouts than Lego objects. CO's low performance on Lego objects in the 3-back condition appears to be an anomaly, as it is much lower than his performance for comparable stimuli in the 1-back and 5-back conditions.

Figure 10b shows the difference in performance between Lego layouts and Lego objects for the critical 1-back and 5-back intervals. At 5-back intervals, $\mathrm{CO}$ was significantly impaired compared to the normal subjects $(\mathrm{z}=-2.50)$, whereas GR's impairment fell just short of significance ( $\mathrm{z}=$ $-1.75)^{4}$.

\footnotetext{
4 In fact, these values may underestimate GR and CO's relative impairment for Lego layouts. In the 5-back condition, 4 of the 10 normal subjects had perfect performance on the Lego layouts, but all scored below ceiling on the Lego objects. Thus, the true advantage for Lego layouts over Lego objects for normal subjects is probably greater than that observed here, in which case GR and CO's lower performance for Lego layouts compared to Lego objects would be even more abnormal. Furthermore, post-hoc analyses revealed that one of the normal subjects showed a difference in performance between Lego layouts and objects that was over 2 SD below the mean of the other nine subjects. When this outlying subject was excluded, $z$-scores were -3.22 for CO and -2.32 for GR.
} 

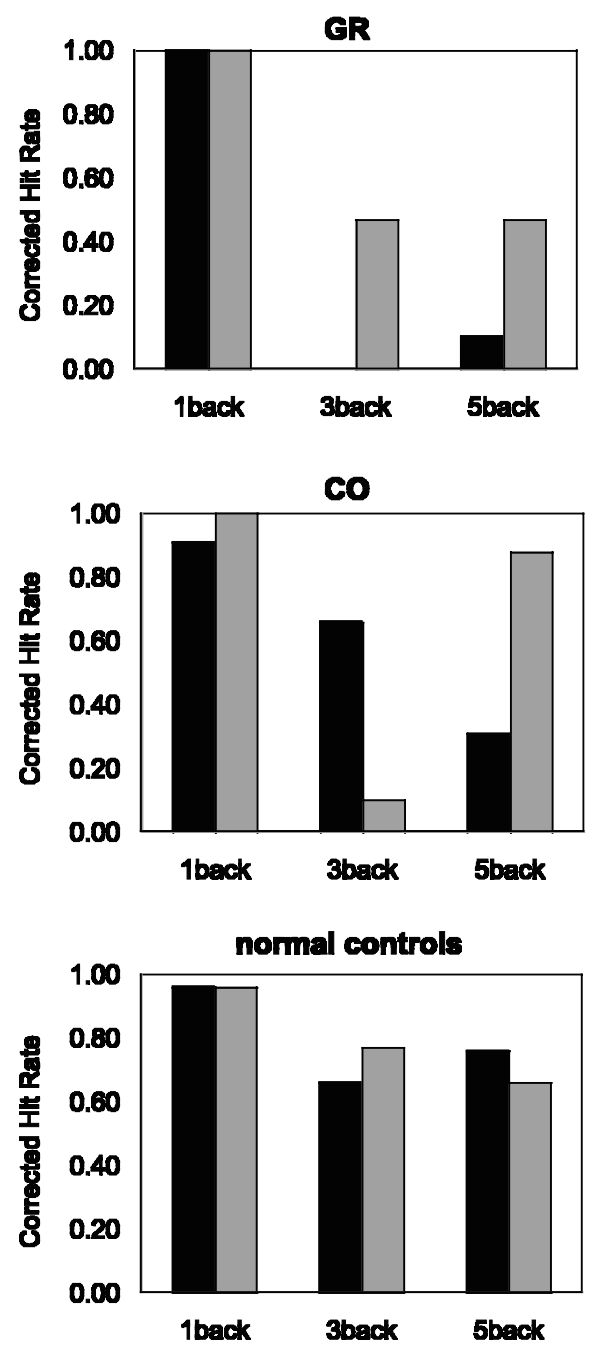

Lego Scenes

\section{Lego Objects}

Figure 10a. Results of Experiment 3, for GR (top) CO (middle) and for 10 age-matched normal controls. Performance measure is proportion of repeated stimuli correctly identified as such, corrected for guessing using the standard formula.

Table 4 shows results for all eight stimulus classes. Although direct comparisons between subjects should be treated with caution since viewing time for different items was not the same for each subject, we do note a few interesting patterns. First of all, there is little or no evidence that GR or CO

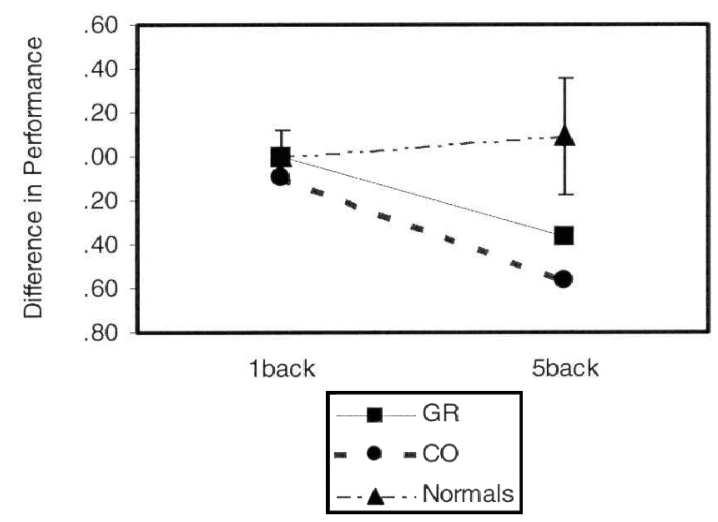

Figure 10b. Difference between Lego layout performance and Lego object performance for the 1-back and 5-back conditions. Error bars indicate one $1 S D$.

show a memory deficit for nonsense words, clay objects, or line drawings of nonsense objects. GR appears to be slightly impaired on faces and houses, but $\mathrm{CO}$ performs normally on these categories. Strikingly, GR shows a severe deficit for the real landscapes, performing more than 40 percentage points lower than normals. Thus, even though there is some evidence that GR might have some impairment for nonscene stimuli, he clearly is much more impaired for scene-like stimuli such as the Lego scenes and landscapes. $\mathrm{CO}$ does not show a similar deficit for the landscapes. However, it should be kept in mind that in one of his two testing sessions, $\mathrm{CO}$ viewed each picture for $3 \mathrm{~s}$, which was much longer than the 1 second used for all nonLego stimuli for GR and normals. Thus, CO's apparently "normal" performance in these conditions might be overestimated.

Taken together, the results of this experiment indicate that GR and $\mathrm{CO}$ are selectively impaired at processing scene-like stimuli. This deficit is consistently apparent at 5-back but not 1-back intervals. This finding suggests that the deficit should be characterised as a memory encoding problem. However, the present results do not allow us to determine whether the difficulty occurs at memory encoding or at subsequent recognition (see Epstein $\&$ Kanwisher, 2001). If GR and CO have a general problem with recognition of topographical materials, then they should not be able to recognise scenes 
Table 4. Full results of Experiment 3: Mean proportion correct (corrected for guessing); SDs in parentheses

\begin{tabular}{llllllllll}
\hline & $\begin{array}{c}\text { Lego } \\
\text { scenes }\end{array}$ & $\begin{array}{l}\text { Land- } \\
\text { scapes }\end{array}$ & Houses & $\begin{array}{c}\text { Lego } \\
\text { objects }\end{array}$ & $\begin{array}{c}\text { Clay } \\
\text { objects }\end{array}$ & $\begin{array}{c}\text { Nonsense } \\
\text { objects }\end{array}$ & $\begin{array}{c}\text { Non- } \\
\text { Words }\end{array}$ & Faces \\
\hline 1-back & GR & 1.00 & 0.94 & 1.00 & 1.00 & 1.00 & 0.94 & 1.00 & 1.00 \\
& CO & 0.91 & 0.85 & 0.91 & 1.00 & 1.00 & 0.79 & 0.74 & 1.00 \\
& Normals & $0.96(0.08)$ & $0.95(0.08)$ & $0.93(0.14)$ & $0.96(0.08)$ & $0.96(0.06)$ & $0.99(0.03)$ & $0.98(0.04)$ & $0.97(0.11)$ \\
& & & & & & & & & \\
3-back & GR & -0.03 & 0.68 & 0.26 & 0.47 & 0.83 & 0.83 & 0.75 & 0.52 \\
& CO & 0.66 & 0.81 & 0.37 & 0.10 & 0.94 & 0.69 & 0.95 & 0.81 \\
& Normals & $0.66(0.32)$ & $0.77(0.21)$ & $0.72(0.15)$ & $0.77(0.20)$ & $0.86(0.16)$ & $0.83(0.15)$ & $0.83(0.17)$ & $0.72(0.20)$ \\
& & & & & & & & & \\
5-back & GR & 0.10 & 0.34 & 0.44 & 0.47 & 0.72 & 0.83 & 0.94 & 0.53 \\
& CO & 0.31 & 0.73 & 0.75 & 0.88 & 0.81 & 0.64 & 1.00 & 0.81 \\
& Normals & $0.76(0.26)$ & $0.77(0.16)$ & $0.68(0.21)$ & $0.66(0.16)$ & $0.91(0.14)$ & $0.83(0.18)$ & $0.93(0.11)$ & $0.78(0.21)$ \\
\hline
\end{tabular}

and landmarks that were learned prior to their injury. We tested this possibility in the next experiment.

\section{Experiment 4: Famous landmarks/faces recognition task}

In this experiment, we examined GR and CO's ability to recognise the visual appearances of places learned prior to injury. Subjects saw a series of famous landmarks intermixed with nonfamous controls. (Imaging experiments have demonstrated that landmarks of this type can activate the PPA almost as strongly as complete scenes; Epstein et al., 1999; Epstein \& Kanwisher, 1998.) They were required to say whether they knew each specific landmark, and to identify the ones they knew. Subjects were instructed to respond affirmatively if they were fairly certain that they recognised the landmark, but not if it only looked generally familiar. For purposes of comparison, subjects also performed an identical test in which the stimuli were famous and nonfamous faces. In both tests, distractor items were designed to be visually and conceptually similar to the target items (see Figure 11). If parahippocampal cortex is required for place recognition, then one might expect GR and $\mathrm{CO}$ to perform poorly compared to normals on the landmark recognition test.

\section{Method}

Each of the two experiments (famous faces, famous landmarks) consisted of 48 trials. Subjects began each trial by pressing the space bar, after which a black-and-white photograph of a face (in the famous faces experiment) or a landmark (in the famous landmarks experiment) appeared on the screen. Subjects were told to press the "j" key if they recognised the specific face or landmark, and the " $\mathrm{f}$ " key if they did not, taking as much time at they needed to make this discrimination but no extra time. Subjects were told to report that they recognised a face or landmark if they felt that they knew it, even if they could not retrieve its name. If they did know the name, they were to say it after pressing the button. Otherwise, they were to say whatever they did remember about the item.

Half of the photographs were of famous and half of nonfamous people and landmarks. The famous faces included public figures (e.g., George Bush, Richard Nixon, Prince Charles) and movie stars (e.g., Harrison Ford, Clark Gable, Marlon Brando) who were prominent prior to the time of GR and CO's injuries; the famous landmarks were buildings and monuments from all over the world (e.g., Eiffel Tower, US Capitol Building, Great Sphinx). The nonfamous stimuli were chosen to match the famous stimuli as closely as possible. Each famous stimulus had a "match." For example, George Bush was matched to a man of similar age in a coat and tie, and the Taj Mahal was matched to a visually 

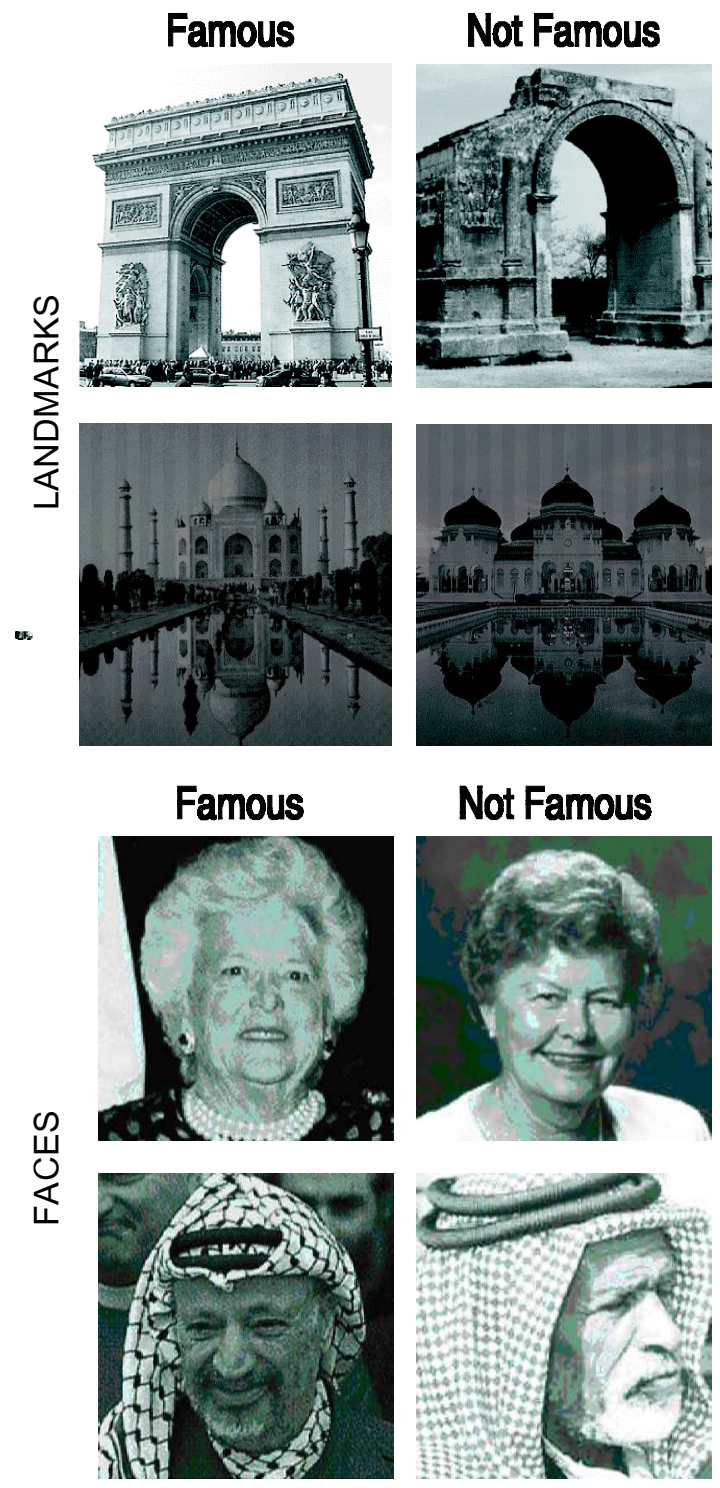

Figure 11. Stimuli for Experiment 4. Each famous landmark (Arc de Triomphe, Taj Mahal) and famous face (Barbara Bush, Yasser Arafat) was matched to a nonfamous building or face that also appeared in the experiment.

similar Asian palace that is not famous (see Figure 11).

\section{Results and discussion}

Results for GR and CO, as well as for five agematched controls, are shown in Figure 12. Both

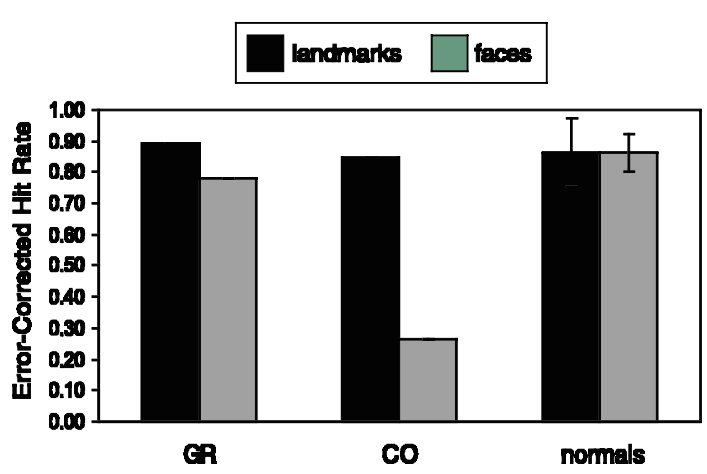

Figure 12. Results of Experiment 4. Performance measure is proportion of famous stimuli reported as "known," corrected for guessing using the standard formula.

GR and CO could recognise the famous landmarks as well normals. In addition, both $\mathrm{GR}$ and $\mathrm{CO}$ were usually able to name the landmarks they successfully identified.

Thus, GR and $\mathrm{CO}$ can recognize landmarks learned prior to injury, demonstrating that at least some of their place recognition abilities are intact. When combined with the fact that GR and CO were able to produce detailed maps of places they lived in prior to their injury (see Case Description), these results suggest that their deficit is primarily in encoding or consolidation of new information rather than in recognition or recall of old information. Note, however, that these results do not prove that their place recognition abilities are entirely normal. GR and CO might suffer from a mild recognition deficit that affects identification of newlylearned stimuli like the Lego layouts in Experiment 3 but not overlearned stimuli like the famous landmarks. Furthermore, it is possible that GR and $\mathrm{CO}$ might be impaired at recognising premorbidly learned places that are defined primarily by their spatial layout even though they are unimpaired at recognising premorbidly learned places that are defined primarily by a single object-like landmark or building (see General Discussion). Nevertheless, the trend of the present evidence is to suggest that GR and $\mathrm{CO}$ have particular problems with novel topographical information.

In the famous-face task, GR's performance was in the normal range. However, he sometimes had difficulty naming the faces even though he clearly 
recognised them. For example, he described Ronald Reagan as "the gipper," Nancy Reagan as "the wife of the gipper," and Clark Gable as "the movie actor who said, 'Scarlett, I don't give a damn'”. CO had a great deal of difficulty identifying the famous faces. This result is not surprising given his extremely low performance on the Benton Face Matching Task. In addition to his topographic problems, $\mathrm{CO}$ appears to suffer from prosopagnosia.

\section{GENERAL DISCUSSION}

We tested two parahippocampal patients (GR and $\mathrm{CO}$ ) on four experiments designed to measure performance as a function of both task (perception, encoding, and recognition) and stimulus type (scenes, objects, faces, and words). The only task that revealed a clear deficit was an n-back recognition memory test (Experiment 3 ). In order to successfully perform this task, subjects had to encode a durable representation of a visual stimulus and use it for later recognition. GR and CO were significantly more impaired on this task when the stimuli were scene-like spatial layouts (i.e., Lego scenes, and real scenes for GR) than when they were objects. No comparable deficits were found in other experiments that tested layout perception (Experiments 1 and 2) and place/landmark recognition (Experiment 4). Taken together, these results indicate that GR and $\mathrm{CO}$ are impaired in their ability to encode novel information about the geometry of surrounding space into memory, but are less impaired at their ability to encode the geometry of novel objects.

We argue that this specific topographical deficit-inability to encode the spatial layout of novel scenes-results from damage to the parahippocampal place area. Although the damage in both patients extends beyond the parahippocampal-lingual territory normally associated with the PPA, this region is clearly lesioned bilaterally in GR and in the right hemisphere in CO. Furthermore, functional imaging demonstrated that GR has no functioning PPA. Thus, the current results and the earlier fMRI work (Epstein et al., 1999;
Epstein \& Kanwisher, 1998) converge to implicate parahippocampal cortex in the encoding of information about scene geometry into memory.

Our assignment of the function of memory encoding to parahippocampal cortex is consistent with the results of earlier case reports of patients with parahippocampal damage who have navigational difficulties in novel but not familiar environments (Habib \& Sirigu, 1987; Ross, 1980; see also Teng \& Squire, 1999). As with GR and CO, these patients were either less impaired or unimpaired on nontopographical memory tasks. Patients have also been described who exhibit the opposite pattern of impairment: an inability to learn faces (sometimes called "prosopamnesia," Cipolotti, Robinson, Blair, \& Frith, 1999; Tippett, Miller, \& Farah, 2000), or faces and verbal materials (Maguire \& Cipolotti, 1998), with an unimpaired ability to learn topographical materials. Taken together, these results argue for a high degree of domain-specificity in the neural systems involved in learning of new information (Gallistel, 1995). The brain may have separate mechanisms for learning faces, places, and words, which can be differentially impaired after damage to specific cortical regions (Cipolotti et al., 1999; Incisa della Rochetta et al., 1996). The present results suggest that the anatomical locus of the mechanism dedicated to learning new topographical information is in the parahippocampal cortex (Aguirre \& D'Esposito, 1999).

In contrast to their impaired ability to encode new topographical information, both GR and CO were able to produce detailed maps of their previous dwellings, and performed normally on the famous landmark recognition test of Experiment 4. These results are generally consistent with Teng and Squire's (1999) report of a patient with extensive medial temporal damage who could recall premorbidly learned but not postmorbidly learned topographical information (see also Ross, 1980). Thus, our results provide further evidence that medial temporal regions such as parahippocampal cortex may be more critical for encoding or consolidating new information into memory than for recall of old information.

One issue that our experiments do not entirely resolve is the precise nature of the topographical 
information encoded by parahippocampal cortex. Clearly, this region is critical for encoding information about the geometry of the currently visible scene even when the "scene" is made out of Lego blocks and therefore has no immediate navigational relevance. However, it is unclear whether this specific deficit can account for all of the real-world navigational difficulties encountered by GR and CO. GR in particular shows such a catastrophic loss of navigational ability that we suspect he is also impaired at encoding the spatial relationships between the current scene and other locations in the world. CO probably also suffers from such difficulties, as he reports that he has trouble remembering how places relate to each other in large-scale space. When he was tested on a simple 3-room videogame environment, he not only was unable to remember the individual rooms (claiming that there were 10 rooms when there were only 3 ), but he also had no memory for the spatial relationships between them. These spatial deficits may be the result of damage to the posterior right hippocampus (in the case of $\mathrm{CO}$ ) or of disconnection of the posterior right hippocampus from the parahippocampal regions that provide much of its cortical input (in both patients; Suzuki \& Amaral, 1994). In either case, the ability to form a cognitive map of new environments like the lab or videogame space could be impaired (Maguire et al., 1998; O'Keefe \& Nadel, 1978). However, it is also possible that parahippocampal cortex itself may be critical for encoding not only the geometry of individual scenes but also the spatial relationships between the locations of those scenes. In this case, the spatial deficits of GR and $\mathrm{CO}$ could be attributed directly to parahippocampal damage rather than to hippocampal damage or disconnection.

The latter scenario would be consistent with Aguirre and D'Esposito's (1999) and Barrash et al.'s (2000) suggestion that parahippocampal cortex is critically involved in combining visual information about the appearance of scenes and landmarks with spatial information about their locations into a unified topographical representation. However, both of these authors argue that the representations of individual scenes (Barrash et al.) and landmarks (Aguirre \& D'Esposito) are supported not by parahippocampal cortex, but by more posterior lingual-fusiform regions. In contrast, we believe that the neuroimaging data indicate that parahippocampal cortex is involved in the encoding of the geometric structure of individual scenes, although it may also play a role in combining these representations with other spatial representations.

Interestingly, GR and $\mathrm{CO}$ were not impaired on recognition of famous landmarks that were mostly buildings. Thus, our data do not preclude the possibility that there may be a separate lingual or fusiform region involved in processing information about buildings (Aguirre, Zarahn, \& D'Esposito, 1998; Chao, Haxby, \& Martin, 1999; Ishai, Ungerleider, Martin, Schouten, \& Haxby, 1999) which might have been preserved in GR and CO despite extensive damage to other regions of the lingual and fusiform gyrus. Alternatively, GR and CO's preserved building recognition abilities might be mediated by interactions between cortical areas that perform shape analysis for any object-like stimulus type (Malach et al., 1995; Kanwisher et al., 1996) and anterior temporal regions in which longterm topographical and semantic memories are stored (McCarthy et al., 1996).

\section{Is perception preserved?}

In contrast to their inability to encode new scenes into memory, GR and $\mathrm{CO}$ have substantially retained their ability to perceive the spatial layout of the currently visible scene (Experiments 1 and 2). GR can follow a route marked on a floor plan, which indicates a good on-line appreciation of his current spatial surroundings. Furthermore, neither GR or CO have any trouble moving through the environment - they move with confidence and do not bump into doors or walls. Problems of the latter type are usually associated with damage to the parietal lobes, which are intact in GR and CO. Thus, at first glance, GR and $\mathrm{CO}$ do not appear to have perceptual impairments (beyond their visual field restrictions).

However, GR does complain of a perceptual deficit, which he describes as an inability to "take in" scenes when they are too complex. Similar reports of an inability to comprehend the 
"globality" of a scene were made by some of the parahippocampally-damaged patients studied by Habib and Sirigu (1987). We were not able to determine the precise nature of this deficit, which may be partly attributable to his visual field restrictions. It appears that his deficit is more salient in the real-world environments than in the picture-perception tasks of Experiments 1 and 2, perhaps because he finds it more difficult to organise visual input when immersed in it than when viewing photographs that subtend only part of the visual field.

We suggest that GR can perceive parts of the scene when he attends to them, but may be unable to integrate information over time into a coherent representation of a scene or place. The fact that GR and $\mathrm{CO}$ are impaired for Lego layouts at 5-back intervals in Experiment 3 is consistent with this hypothesis. The time between the first and second presentations of a stimulus is very short in this case (and even shorter with 3-back intervals, where GR also shows evidence of a deficit). In both patients, the representation of layout information formed by perception of a Lego scene must either have an abnormally short persistence, be abnormally susceptible to interference, or both. If so, they might find it impossible to sustain a spatial frame or schema long enough to use it to organise information obtained from many different glances at a complex scene (Intraub, 1997). The result would be an inability to understand the "globality" of a scene, even though all the details were perfectly perceived.

\section{Non-topographical mnemonic abilities}

Although not as severe as their topographical memory problems, GR and $\mathrm{CO}$ do have some memory difficulties in other domains. For example, both performed poorly on the Rey AVLT, which required them to remember a list of unrelated words. Both also report some subtle episodic memory problems (see Case Description). CO's problems could partly result from damage to the right hippocampus - indeed, $\mathrm{CO}$ appeared to have the more acute episodic memory problems. However, GR has no hippocampal damage, so the fact that he displays episodic memory problems suggests that parahippocampal cortex is involved in the forma- tion of episodic memories (cf., Wagner et al., 1998).

In previous work (Epstein et al., 1999), we argued that spatial context information encoded by parahippocampal cortex might play a role in establishing event memories by encoding information about where events take place. The inability to encode this information might cause problems in tasks such as the Rey AVLT, where one has to recall a list of unrelated words: If spatial (and perhaps temporal) context information is not encoded, it will be harder subsequently to recall the distinct event of hearing each word (Tulving, 1983). In contrast, lack of spatial context information would be less of a problem in tasks where one is only required to recognise previously encountered verbal materials but not to reproduce the spatiotemporal context in which they were experienced. Indeed, GR and CO performed as well as normals on the nonsense words in Experiment 4, where recognition memory but not episodic memory was required for successful performance. Interestingly, GR and $\mathrm{CO}$ also did relatively well on the Logical Memory subtest of the WMS-III, perhaps because the narrative framework of a story provides sufficient contextual information to overcome the absence of spatial context and mediate successful verbal retrieval.

\section{Differences between GR and CO}

Although we have discussed GR and CO together, there are a number of relevant differences between them. One open question is whether these differences can be attributed to the different effects of unilateral and bilateral parahippocampal damage. In general, GR's topographical difficulties were more severe than CO's. CO did surprisingly well on the Warrington Topographical Memory Test, in which he was required to remember detailed realworld city scenes, and also performed substantially better on real scenes than Lego scenes in Experiment 3. Insofar as $\mathrm{CO}$ has intact left parahippocampal cortex, this suggests that the left PPA might be sufficient to encode a certain amount of (perhaps semantic) information about real-world scenes, but the right PPA is necessary to encode 
Lego scenes that must be recognised solely on the basis of their spatial structure. Clearly, however, the amount of topographical information that can be encoded using only a left PPA is strictly limited. Another difference between the two patients is that GR reports some kind of perceptual problem, but $\mathrm{CO}$ does not. It is presently unclear whether these differences are indicative of qualitatively different kinds of impairment after unilateral and bilateral lesions, or whether the same abilities are affected in both cases but to a more severe degree with bilateral lesions.

\section{Conclusion}

We examined two patients with lesions to parahippocampal cortex. Both exhibited severe topographical difficulties, particularly in new environments. A key component of their problem is a memory encoding deficit that is more severe for visual stimuli that depict spaces that one can navigate through or act within than for other visual materials. We found no clear evidence that parahippocampal cortex is necessary for the initial perceptual processing of visual scenes, recognition of landmarks, or recall of premorbidly learned topographical information. Parahippocampal cortex may be the neuroanatomical locus of a learning mechanism preferentially involved in the encoding of topographical materials into memory.

Manuscript received 20 December 1999 Revised manuscript received 14 June 2000 Revised manuscript accepted 20 October 2000

\section{REFERENCES}

Aguirre G.K., \& D’Esposito, M. (1999). Topographical disorientation: A synthesis and taxonomy. Brain, 122, 1613-1628.

Aguirre, G.K., Detre, J.A., Alsop, D.C., \& D'Esposito, M. (1996). The parahippocampus subserves topographical learning in man. Cerebral Cortex, 6, 823829.

Aguirre, G.K., Zarahn, E., \& D’Esposito, M. (1998).An area within human ventral cortex sensitive to "build- ing" stimuli: Evidence and implications. Neuron, 21, 373-383.

Barrash, J., Damasio, H., Adolphs, R., \& Tranel, D. (2000). The neuroanatomical correlates of route learning impairment. Neuropsychologia, 38, 820-836.

Beauchamp, M.S., Haxby, J.V., Rosen, A.C., \& DeYoe, E.A. (2000). Functional MRI correlates of recovery of function in acquired cerebral dyschromatopsia. Neuropsychologia, 38, 1170-1179.

Bohbot, V., Kalina, M., Stepaknova, K., Spackova, N., Petrides, M., \& Nadel, L. (1998). Spatial memory deficits in patients with lesions to the right hippocampus and to the right parahippocampal cortex. Neuropsychologia, 36, 1217-1238.

Chao, L.L., Haxby, J.V., \& Martin, A. (1999). Attribute-based neural substrates in temporal cortex for perceiving and knowing about objects. Nature Neuroscience, 2, 913-919.

Chen, L.L., Lin, L.H., Green, E.J., Barnes, C.A., \& McNaughton, B.L. (1994). Head direction cells in the rat posterior cortex, I. Anatomical distribution and behavioral modulation. Experimental Brain Research, 101, 24-34.

Cheng, K. (1986). A purely geometric module in the rat's spatial representation. Cognition, 23, 149-178.

Cipolotti, L., Robinson, G., Blair, J., \& Frith, U. (1999). Fractionation of visual memory: Evidence from a case with multiple neurodevelopmental impairments. Neuropsychologia, 37, 455-465.

Eichenbaum, H., Dudchenko, P., Wood, E., Shapiro, M., \& Tanila, H. (1999). The hippocampus, memory, and place cells: Is it spatial memory or a memory space? Neuron, 23, 209-226.

Epstein, R., Harris, A., Stanley, D., \& Kanwisher, N. (1999). The parahippocampal place area: Recognition, navigation, or encoding? Neuron, 23, 115-125.

Epstein, R., \& Kanwisher, N. (1998). A cortical representation of the local visual environment. Nature, 392, 598-601.

Epstein, R., \& Kanwisher, N. (2001). Mnemonic functions of parabippocampal cortex: An event-related fMRI study. Manuscript submitted for publication.

Evans, A.C., Collins, D.L., Mills, S.R., Brown, E.D., Kelly, R.L., \& Peters, T.M. (1993). 3D statistical neuroanatomical models from $305 \mathrm{MRI}$ volumes. In Proceedings of the IEEE-Nuclear Science Symposium and Medical Imaging Conference (pp. 1813-1817).

Gallistel, C.R. (1990). The organization of learning. Cambridge, MA: MIT Press.

Gallistel, C.R. (1995). The replacement of general-purpose theories with adaptive specializations. In M.S. 
Gazzaniga (Ed.), The cognitive neurosciences. Cambridge, MA: MIT Press.

Ghaem, O., Mellet, E., Crivello, F., Tzourio, N., Mazoyer, B., Berthoz, A., \&Denis, M. (1997). Mental navigation along memorized routes activates the hippocampus, precuneus, and insula. NeuroReport, 8 , 739-744.

Habib, M., \& Sirigu, A. (1987). Pure topographical disorientation: a definition and anatomical basis. Cortex, 23, 73-85.

Hecaen, H., Tzortzis, C., \& Rondot, P. (1980). Loss of topographic memory with learning deficits. Cortex, 16, 525-542.

Hermer, L., \& Spelke, E.S. (1994). A geometric process for spatial reorientation in young children. Nature, 370, 57-59.

Hermer, L., \& Spelke, E.S. (1996). Modularity and development: the case of spatial reorientation. Cognition, 61, 195-232.

Hermer-Vasquez, L., Spelke, E.S., \& Katsnelson, A.S. (1999). Sources of flexibility in human cognition: Dual-task studies of space and language. Cognitive Psychology, 39, 3-36.

Incisa della Rocchetta, A., Cipolotti, L., \&Warrington, E.K. (1996). Topographical disorientation: Selective impairment of locomotor space? Cortex, 32, 727-735.

Intraub, H. (1997). The representation of visual scenes. Trends in Cognitive Sciences, 1, 217-222.

Ishai, A., Ungerleider, L.G., Martin, A., Schouten, J.L., \& Haxby, J.V. (1999). Distributed representation of objects in the human ventral visual pathway. Proceedings of the National Academy of Sciences, USA, 96, 9379-9384.

Kanwisher, N., Chun, M.M., McDermott, J., \& Ledden, P.J. (1996). Functional imaging of human visual recognition. Cognitive Brain Research, 5, 55-67.

Kanwisher, N., McDermott, J., \& Chun, M.M. (1997). The fusiform face area: a module in human extrastriate cortex specialized for face perception. Journal of Neuroscience, 17, 4302-4311.

Landis, T., Cummings, J.L., Benson, D.F., \& Palmer, E.P. (1986). Loss of topographic familiarity: an environmental agnosia. Archives of Neurology, 43, 132136.

Levine, D.N., Warach, J., \& Farah, M.J. (1985). Two visual systems in mental imagery: Dissociation of "what" and "where" in imagery disorders due to bilateral posterior cerebral lesions. Neurology, 35, 10101018.

Maguire, E.A., Burgess, N., Donnett, J.G., Frackowiak, R.S.J., Frith, C.D., \& O'Keefe, J. (1998). Knowing where and getting there: A human navigation network. Science, 280, 921-924.

Maguire, E.A., Burke, T., Philips, J., \& Staunton, H. (1996). Topographical disorientation following unilateral temporal lobe lesions in humans. Neuropsychologia, 34, 994-1001.

Maguire, E.A., \& Cipolotti, L. (1998). Selective sparing of topographical memory. Journal of Neurology, Neurosurgery and Psychiatry, 65, 903-909.

Maguire, E.A., Frackowiak, R.S.J., \& Frith, C.D. (1996). Learning to find your way: A role for the human hippocampal region. Proceedings of the Royal Society of London B Series [Biological Sciences], 263, 1745-1750.

Maguire, E.A., Frackowiak, R.S.J., \& Frith, C.D. (1997). Recalling routes around London: Activation of the right hippocampus in taxi drivers. Journal of Neuroscience, 17, 7103-7110.

Malach, R., Reppas, J.B., Benson, R.B., Kwong, K.K., Jiang, H., Kennedy, W.A., Ledden, P.J., Brady, T.J., Rosen, B.R., \& Tootell, R.B.H. (1995). Objectrelated activity revealed by functional magnetic resonance imaging in human occipital cortex. Proceedings of the National Academy of Science, USA, 92, 81358138.

Margules, J., \& Gallistel, C.R. (1988). Heading in the rat: determination by environmental shape. Animal Learning and Behavior, 16, 404-410.

McCarthy, R.A., Evans, J.J., \& Hodges, J.R. (1996). Topographic amnesia: Spatial memory disorder, perceptual disfunction, or category-specific semantic memory impairment. Journal of Neurology, Neurosurgery and Psychiatry, 60, 318-325.

Milner, A.D., \& Goodale, M.A. (1995). The visual brain in action. Oxford: Oxford University Press.

O'Keefe, J., \& Nadel, L. (1978). The hippocampus as a cognitive map. Oxford: Oxford University Press.

Pallis, C.A. (1955). Impaired identification of faces and places with agnosia for colours. Journal of Neurology, Neurosurgery and Psychiatry, 18, 218-224.

Pigott, S., \& Milner, B. (1993). Memory for different aspects of complex visual scenes after unilateral temporal- or frontal-lobe resection. Neuropsychologia, 31, $1-15$.

Rao, S.M., Hammeke, T.A., McQuillen, M.P., Khatri, B.O., \& Lloyd, D. (1984). Memory disturbance in chronic progressive multiple sclerosis. Archives of Neurology, 41, 625-631.

Ross, E.D. (1980). Sensory-specific and fractional disorders of recent memory in man: I. Isolated loss of visual recent memory. Archives of Neurology, 37, 193-200. 
Spreen, O., \& Strauss, E. (1998). A compendium of neuropsychological tests: Administration, norms, and commentary. New York: Oxford University Press.

Stark, M., Coslett, H.B., \& Saffran, E.M. (1996). Impairment of an egocentric map of locations: Implications for perception and action. Cognitive Neuropsychology, 13, 481-523.

Suzuki, W.A., \& Amaral, D.G. (1994). Perirhinal and parahippocampal cortices of the macaque monkey: Cortical referents. Journal of Comparative Neurology, 350, 497-533.

Talairach J., \& Tournoux, P. (1988). Co-planar stereotactic atlas of the human brain. Stuttgart, Germany: Thieme.

Teng, E., \& Squire, L.R. (1999). Memory for places learned long ago is intact after hippocampal damage. Nature, 400, 675-677.

Tippett, L.J., Miller, L.A., \& Farah, M.J. (2000). Prosopamnesia: A selective impairment in face learning. Cognitive Neuropsychology, 17, 241-256.
Tulving, E. (1983). Elements of episodic memory. Oxford: Clarendon Press.

Wagner, A.D., Schacter, D.L., Rotte, M., Koutstaal, W., Maril, A., Dale, A.M., Rosen, B.R., \& Buckner, R.L. (1998). Building memories: Remembering and forgetting of verbal experiences as predicted by brain activity. Science, 281, 1188-1191.

Wechsler, D. (1997a). Wecbsler Adult Intelligence ScaleIII: Administration and scoring manual. San Antonio, TX: The Psychological Corporation, Harcourt Brace \& Company.

Wechsler, D. (1997b). Wechsler Memory Scale-III: Administration and scoring manual. San Antonio, TX: The Psychological Corporation, Harcourt Brace \& Company.

Whiteley, A.M., \& Warrington, E.K. (1978). Selective impairment of topographic memory: A single case study. Journal of Neurology, Neurosurgery and Psychiatry, 41, 575-578. 